\title{
Positivity for equations involving polyharmonic operators with Dirichlet boundary conditions
}

\section{Hans-Christoph Grunau ${ }^{1}$, Guido Sweers ${ }^{2}$}

1 Mathematisches Institut, Universität Bayreuth, D-95440 Bayreuth, Germany

2 Vakgroep Algemene Wiskunde, Technische Universiteit Delft, Postbus 5031, 2600 GA Delft Netherlands

Received: 13 April 1995 /Revised version: 6 March 1996

Mathematics Subject Classification (1991): 35J40; 35B50.

\section{Introduction}

Cranston, Fabes and Zhao ([26], [5]) established the uniform bound

$$
\sup _{\substack{x, y \in \Omega \\ x \neq y}} \frac{\int_{\Omega} G_{1, n}(x, z) G_{1, n}(z, y) d z}{G_{1, n}(x, y)} \leq M<\infty,
$$

where $G_{1, n}(x, y)$ is the Green function for the Laplacian $-\Delta$ with Dirichlet boundary conditions on a Lipschitz domain $\Omega \subset \mathbb{R}^{n}$ with $n \geq 3$ (see [27] for $n=2$ ). This estimate was used in [23] and [18] to obtain positivity, uniformly with respect to $f \geq 0$, for noncooperative elliptic systems as:

$$
\left\{\begin{array}{rlrl}
-\Delta u=f-\varepsilon v & & \text { in } \Omega, \\
-\Delta v & =u & & \text { in } \Omega, \\
u=v & =0 & & \text { on } \partial \Omega
\end{array}\right.
$$

when $\varepsilon \geq 0$ is small. In particular, $\Delta^{2} v+\varepsilon v \geq 0$ in $\Omega$, with $v=\Delta v=0$ on $\partial \Omega$, implies $v \geq 0$ for $\varepsilon$ small. In numerical experiments ([14]) for one dimension a similar behaviour was observed under Dirichlet boundary conditions $v=\frac{\partial}{\partial n} v=0$.

In this paper we will derive a $3-G$ type theorem as in (1) but with $G_{1, n}$ replaced by the Green function $G_{m, n}$ for the $m$-polyharmonic operator with Dirichlet boundary conditions and with $\Omega$ replaced by the unit ball $B$ in $\mathbb{R}^{n}$ with $n \geq 1$ :

$$
\left\{\begin{aligned}
(-\Delta)^{m} u & =f \quad \text { in } B \\
\mathcal{D}_{m} u & =0 \text { on } \partial B
\end{aligned}\right.
$$

where

$$
\mathcal{D}_{m} u=\left(D^{k} u\right)_{k \in \mathbb{N}^{n},|k| \leq m-1} .
$$


We use a multi-index $k \in \mathbb{N}^{n}$ with $D^{k}=\left(\frac{\partial}{\partial x_{1}}\right)^{k_{1}} \ldots\left(\frac{\partial}{\partial x_{n}}\right)^{k_{n}}$ and $|k|=k_{1}+\ldots+k_{n}$. $\mathbb{N}$ is the set of nonnegative integers. Notice that the Dirichlet boundary condition may also be written as

$$
\left(u, \frac{\partial}{\partial r} u, \frac{\partial^{2}}{\partial r^{2}} u, \ldots, \frac{\partial^{m-1}}{\partial r^{m-1}} u\right)=0 \text { on } \partial B .
$$

Boggio in [4] (see also [9], [10]) showed positivity of $G_{m, n}$ on the ball $B$. Here we will establish two-sided pointwise estimates for $G_{m, n}$ and estimates from above for derivatives of $G_{m, n}$. These estimates enable us to prove 3-G Theorem type results.

For these results we will only use the ball in $\mathbb{R}^{n}$ as a domain. In contrary to the Laplacian the Green function for the biharmonic or polyharmonic operator is not positive for arbitrary domains. Hence the estimate of $G_{m, n}$ from below (by a positive function) necessarily restricts the possible domains. The question whether or not the estimates from above remain true on arbitrary smooth and bounded domains, is open as far as the authors know. Counterexamples of sign changing superpolyharmonic functions can be found in [6], [7, p. 275], [17] and [22].

The $3-\mathrm{G}$ results are used in three directions. First, one can prove that on $B$ also the inverse of polyharmonic operators that are perturbed by small lower order terms, are positivity preserving. That is, if the coefficients $a_{k}$ in

$$
(-\Delta)^{m} u+\sum_{|k|<2 m} a_{k}(\cdot) D^{k} u=f,
$$

are sufficiently small, $f$ positive implies that the solution $u$ of the Dirichlet problem for (3) is positive. Secondly, as in [24], we obtain positivity results for systems of these operators. Thirdly, estimates for these Green functions on the unit ball in $\mathbb{R}^{n}$ can be used to obtain local maximum principles for solutions of differential inequalities in arbitrary domains. Results in this last direction will be studied in a forthcoming paper [11]. As a consequence we will show in [11] existence of classical solutions of higher order semilinear elliptic equations for a much wider class as in [9]-[10].

\section{Estimates for $G_{m, n}$}

We will use the following notations.

i. $B=\left\{x \in \mathbb{R}^{n} ;|x|<1\right\}$ with $n \geq 1$.

ii. $s \wedge t=\min (s, t)$ and $s^{+}=\max (s, 0)$ for $s, t \in \mathbb{R}$.

iii. $\mathcal{G}_{m, n}$ is the Green operator for $(2)$, that is, $u=\mathcal{G}_{m, n} f$ with

$$
\left(\mathcal{G}_{m, n} f\right)(x)=\int_{B} G_{m, n}(x, y) f(y) d y
$$

iv. For $x, y \in B$ :

$$
\begin{aligned}
{[x y] } & =|x-y|, \\
{[X Y] } & =|| x\left|y-\frac{x}{|x|}\right|, \\
d(x) & =1-|x|, \\
\Theta(x, y) & =[X Y]^{2}-[x y]^{2} .
\end{aligned}
$$


Note that $[X Y]=\sqrt{|x|^{2}|y|^{2}-2 x \cdot y+1}=[Y X]$ and that $d(x)$ is the distance of $x$ to the boundary. Moreover

$$
\Theta(x, y)=\left(1-|x|^{2}\right)\left(1-|y|^{2}\right) .
$$

Hence $[X Y]>[x y]$ for $x, y \in B$.

Lemma 2.1 (Boggio) [4, p. 126] The Green function $G_{m, n}$ is as follows:

$$
G_{m, n}(x, y)=k_{m, n}|x-y|^{2 m-n} \int_{1}^{[X Y] /[x y]} \frac{\left(v^{2}-1\right)^{m-1}}{v^{n-1}} d v
$$

with $k_{m, n}$ some fixed positive constants.

Remark 1. For $m=2$ one finds

$$
\begin{aligned}
& G_{2, n}(x, y)= \\
& =c_{n}\left\{\begin{array}{cc}
{[x y]^{3}-[X Y]^{3}+\frac{3}{2} \Theta(x, y)[X Y]} & \text { for } n=1, \\
{[x y]^{2}(2 \log [x y]-2 \log [X Y])+\Theta(x, y)} & \text { for } n=2, \\
-[x y]+[X Y]-\frac{1}{2} \Theta(x, y)[X Y]^{-1} & \text { for } n=3, \\
-2 \log [x y]+2 \log [X Y]-\Theta(x, y)[X Y]^{-2} & \text { for } n=4, \\
{[x y]^{-n+4}-[X Y]^{-n+4}-\frac{n-4}{2} \Theta(x, y)[X Y]^{-n+2} \text { for } n \geq 5,}
\end{array}\right.
\end{aligned}
$$

with

$$
\begin{array}{ll}
c_{n}=\left(2|(n-4)(n-2)| S_{n}\right)^{-1} & \text { for all } n \notin\{2,4\}, \\
c_{n}=\quad\left(8 S_{n}\right)^{-1} & \text { for } n \in\{2,4\} .
\end{array}
$$

$S_{n}$ is the area of the surface of the unit ball in $\mathbb{R}^{n}\left(S_{1}=2\right)$.

Starting from the explicit formula we will prove a two-sided estimate. But to simplify the statements we will first define an ordering and a related equivalency relation for nonnegative functions.

Definition 2.2 Let $f, g \geq 0$. We call $f \sim g$ on $\Omega$, iff there are $c_{1}, c_{2}>0$ such that

$$
c_{1} g(x) \leq f(x) \leq c_{2} g(x) \quad \text { for all } x \in \Omega .
$$

We will say $f \preceq g$ on $\Omega$, iff there is $c>0$ such that

$$
f(x) \leq \operatorname{cg}(x) \quad \text { for all } x \in \Omega .
$$

Proposition 2.3 On $B^{2}$ (that is $(x, y) \in B^{2}$ ) we have the following. 
i. For $2 m<n$ :

$$
G_{m, n}(x, y) \sim|x-y|^{2 m-n}\left(1 \wedge \frac{d(x)^{m} d(y)^{m}}{|x-y|^{2 m}}\right) .
$$

ii. For $2 m=n$ :

$$
G_{m, n}(x, y) \sim \log \left(1+\frac{d(x)^{m} d(y)^{m}}{|x-y|^{2 m}}\right)
$$

iii. For $2 m>n$ :

$$
G_{m, n}(x, y) \sim(d(x) d(y))^{m-\frac{1}{2} n}\left(1 \wedge \frac{d(x)^{\frac{1}{2} n} d(y)^{\frac{1}{2} n}}{|x-y|^{n}}\right) .
$$

Proposition 2.4 Let $k \in \mathbb{N}^{n}$ with $|k| \leq 2 m$. Then on $B^{2}$ we have the following.

i. For $|k| \geq 2 m-n$ and $n$ odd, or, $|k|>2 m-n$ and $n$ even:

(a) if $|k| \leq m$ then

$$
\left|D_{x}^{k} G_{m, n}(x, y)\right| \preceq|x-y|^{2 m-n-|k|}\left(1 \wedge \frac{d(x)^{m-|k|} d(y)^{m}}{|x-y|^{2 m-|k|}}\right) ;
$$

(b) if $|k| \geq m$ then

$$
\left|D_{x}^{k} G_{m, n}(x, y)\right| \preceq|x-y|^{2 m-n-|k|}\left(1 \wedge \frac{d(y)^{m}}{|x-y|^{m}}\right) .
$$

ii. For $|k|=2 m-n$ and $n$ even:

(a) if $|k| \leq m$ (that is $m \leq n$ ) then

$$
\left|D_{x}^{k} G_{m, n}(x, y)\right| \preceq \log \left(2+\frac{d(y)}{|x-y|}\right)\left(1 \wedge \frac{d(x)^{m-|k|} d(y)^{m}}{|x-y|^{2 m-|k|}}\right) ;
$$

(b) if $|k| \geq m$ (that is $m \geq n$ ) then

$$
\left|D_{x}^{k} G_{m, n}(x, y)\right| \preceq \log \left(2+\frac{d(y)}{|x-y|}\right)\left(1 \wedge \frac{d(y)^{m}}{|x-y|^{m}}\right) .
$$

iii. For $|k| \leq 2 m-n$ and $n$ odd, or, $|k|<2 m-n$ and $n$ even:

(a) if $|k| \leq m-\frac{1}{2} n$ then

$$
\left|D_{x}^{k} G_{m, n}(x, y)\right| \preceq d(x)^{m-\frac{1}{2} n-|k|} d(y)^{m-\frac{1}{2} n}\left(1 \wedge \frac{d(x)^{\frac{1}{2} n} d(y)^{\frac{1}{2} n}}{|x-y|^{n}}\right) ;
$$

(b) if $m-\frac{1}{2} n \leq|k| \leq m$ then

$$
\left|D_{x}^{k} G_{m, n}(x, y)\right| \preceq d(y)^{2 m-n-|k|}\left(1 \wedge \frac{d(x)^{m-|k|} d(y)^{n-m+|k|}}{|x-y|^{n}}\right) ;
$$


(c) if $m \leq|k|$ then

$$
\left|D_{x}^{k} G_{m, n}(x, y)\right| \preceq d(y)^{2 m-n-|k|}\left(1 \wedge \frac{d(y)^{n-m+|k|}}{|x-y|^{n-m+|k|}}\right) .
$$

Remark 2. The Green function for the Laplacian $(n>2, m=1)$ satisfies the estimates above on arbitrary bounded domains with $C^{2, \gamma}$-smooth boundary. This result (among others) was established by K. O. Widman in [25].

Remark 3. For $p, q \geq 0$ one finds on $B^{2}$

$$
\left(1 \wedge \frac{d(x)^{p} d(y)^{q}}{|x-y|^{p+q}}\right) \sim\left(1 \wedge \frac{d(x)}{|x-y|}\right)^{p}\left(1 \wedge \frac{d(y)}{|x-y|}\right)^{q},
$$

and if $p+q>0$

$$
\log \left(1+\frac{d(x)^{p} d(y)^{q}}{|x-y|^{p+q}}\right) \sim \log \left(2+\frac{d(y)}{|x-y|}\right)\left(1 \wedge \frac{d(x)^{p} d(y)^{q}}{|x-y|^{p+q}}\right) .
$$

These results follow from Lemma 3.2 viii. and ix.

Remark 4. From Lemma 3.3 it follows that for all three cases in Proposition 2.4 iii. we have

$$
\left|D_{x}^{k} G_{m, n}(x, y)\right| \preceq d(x)^{m-\frac{1}{2} n-|k|} d(y)^{m-\frac{1}{2} n}\left(1 \wedge \frac{d(x)^{\frac{1}{2} n} d(y)^{\frac{1}{2} n}}{|x-y|^{n}}\right) .
$$

In the case that $m-\frac{1}{2} n \leq|k| \leq m$ both estimates (Prop. 2.4 iii.(b) and (8)) are equivalent as can be seen from Lemma 3.3 ii. When $|k|>m$ the estimate in (8) is less sharp as the one in Prop. 2.4 iii. (c).

\section{Proving the Green function estimates}

We will repeatedly distinguish the cases $[x y] \geq \frac{1}{2}[X Y]$ and $[x y] \leq \frac{1}{2}[X Y]$. The following Lemma will help us doing so.

Lemma 3.1 If $[x y] \geq \frac{1}{2}[X Y]$, then

$$
\begin{gathered}
d(x) d(y) \leq 3|x-y|^{2}, \\
\max (d(x), d(y)) \leq 3|x-y| .
\end{gathered}
$$

If $[x y] \leq \frac{1}{2}[X Y]$, then

$$
\begin{gathered}
\frac{3}{4}|x-y|^{2} \leq \frac{3}{16}[X Y]^{2} \leq d(x) d(y), \\
\frac{1}{4} d(x) \leq d(y) \leq 4 d(x) .
\end{gathered}
$$


Proof. If $[x y] \geq \frac{1}{2}[X Y]$, then $d(x) d(y) \leq[X Y]^{2}-[x y]^{2} \leq \frac{3}{4}[X Y]^{2}$ which implies (9). The estimate in (10) follows from

$$
\begin{gathered}
d(x)^{2} \leq d(x)(d(y)+|x-y|) \leq \\
\leq 3|x-y|^{2}+d(x)|x-y| \leq \\
\leq 4|x-y|^{2}+\frac{1}{4} d(x)^{2}
\end{gathered}
$$

and a similar estimate for $d(y)$.

If $[x y] \leq \frac{1}{2}[X Y]$, then $d(x) d(y) \geq \frac{1}{4}\left([X Y]^{2}-[x y]^{2}\right) \geq \frac{3}{16}[X Y]^{2}$ which implies (11) and moreover

$$
\begin{aligned}
& d(x) \leq d(y)+|x-y| \leq d(y)+\sqrt{\frac{4}{3} d(x) d(y)} \leq \\
& \leq d(y)+\frac{1}{\sqrt{3}}(d(x)+d(y)) \leq \frac{3}{5} d(x)+\frac{8}{5} d(y)
\end{aligned}
$$

implies $d(x) \leq 4 d(y)$. Similarly $d(y) \leq 4 d(x)$ and (12) follows.

The following equivalencies will be used several times.

Lemma 3.2 On $\mathbb{R}^{+} \times \mathbb{R}^{+}$(for $s, t \in \mathbb{R}^{+}$) we have:

$$
\begin{aligned}
\text { i. } & \min \left(\frac{1}{s}, \frac{1}{t}\right) \\
\text { ii. } \quad \max (s, t) & \sim s+t .
\end{aligned}
$$

On $B^{2}$ (for $x, y \in B$ ) we have with $p, q \geq 0$ fixed:

$$
\begin{array}{rlrl}
\text { iii. } & \text { iv. } & r(x, y) & \sim d(x) d(y), \\
\text { iv. } & 1 \wedge \frac{d(y)}{|x-y|} & \sim 1 \wedge \frac{d(y)}{d(x)} \wedge \frac{d(y)}{|x-y|}, \\
\text { vi. } & 1 \wedge \frac{d(x) d(y)}{|x-y|^{2}} & \sim \frac{d(y)}{d(x)} \wedge \frac{d(x)}{d(y)} \wedge \frac{d(x) d(y)}{|x-y|^{2}}, \\
\text { vii. } & 1 \wedge \frac{d(x)^{p} d(y)^{q}}{|x-y|^{p+q}} & \sim 1 \wedge \frac{d(x)^{p}}{|x-y|^{p}} \wedge \frac{d(y)^{q}}{|x-y|^{q}} \wedge \frac{d(x)^{p} d(y)^{q}}{|x-y|^{p+q}}, \\
\text { viii. } & 1 \wedge \frac{d(x)^{p} d(y)^{q}}{|x-y|^{p+q}} & \sim\left(1 \wedge \frac{d(x)}{|x-y|}\right)^{p}\left(1 \wedge \frac{d(y)}{|x-y|}\right)^{q} .
\end{array}
$$

On $B^{2}$ (for $x, y \in B$ ) we have with $p, q \geq 0$ and $p+q>0$ fixed:

$$
i x . \log \left(1+\frac{d(x)^{p} d(y)^{q}}{|x-y|^{p+q}}\right) \sim \log \left(2+\frac{d(y)}{|x-y|}\right)\left(1 \wedge \frac{d(x)^{p} d(y)^{q}}{|x-y|^{p+q}}\right) .
$$

Remark 1. The statements in viii., ix. imply $(6,7)$ in the remark following Proposition 2.4 .

Proof. 
i. and ii. are straightforward; iii. follows from (4).

iv. For the first step we use

$$
[X Y]^{2}=|x|^{2}|y|^{2}-2 x \cdot y+1 \geq(1-|x||y|)^{2} \geq(1-|x|)^{2}=d(x)^{2} .
$$

With a similar estimate by $d(y)$ and with (4) we find left $\geq \frac{1}{3}$ right. By (4) we also find

$$
\begin{gathered}
{[X Y] \leq \sqrt{|x-y|^{2}+4 d(x) d(y)} \leq} \\
\leq|x-y|+2 \sqrt{d(x) d(y)} \leq|x-y|+d(x)+d(y) .
\end{gathered}
$$

v. We use Lemma 3.1 to find the following. If $[x y] \geq \frac{1}{2}[X Y]$, then $\frac{d(y)}{|x-y|} \preceq \frac{d(y)}{d(x)}$ and if $[x y] \leq \frac{1}{2}[X Y]$, then $1 \preceq \frac{d(y)}{d(x)}$.

vi. The sixth equivalency is proven as follows. Since $\min \left(t, t^{-1}\right) \leq 1$ on $\mathbb{R}^{+}$, one finds left $\succeq$ right. As in v. we find by Lemma 3.1 if $[x y] \geq \frac{1}{2}[X Y]$, then

$$
\frac{d(x) d(y)}{|x-y|^{2}} \preceq \frac{d(x) d(y)}{d(x)^{2}} \quad \text { and } \quad \frac{d(x) d(y)}{|x-y|^{2}} \preceq \frac{d(x) d(y)}{d(y)^{2}},
$$

and if $[x y] \leq \frac{1}{2}[X Y]$, then

$$
1 \preceq \frac{d(y)}{d(x)} \quad \text { and } \quad 1 \preceq \frac{d(x)}{d(y)} .
$$

vii. By Lemma 3.1 if $[x y] \geq \frac{1}{2}[X Y]$ then

$$
\frac{d(x)^{p}}{|x-y|^{p}} \wedge \frac{d(y)^{q}}{|x-y|^{q}} \succeq \frac{d(x)^{p} d(y)^{q}}{|x-y|^{p+q}},
$$

and if $[x y] \leq \frac{1}{2}[X Y]$ then

$$
\frac{d(x)^{p}}{|x-y|^{p}} \wedge \frac{d(y)^{q}}{|x-y|^{q}} \succeq 1 .
$$

viii. One uses again Lemma 3.1. If $[x y] \geq \frac{1}{2}[X Y]$ then

$$
\left(1 \wedge \frac{d(x)^{p} d(y)^{q}}{|x-y|^{p+q}}\right) \sim \frac{d(x)^{p} d(y)^{q}}{|x-y|^{p+q}} \sim\left(1 \wedge \frac{d(x)}{|x-y|}\right)^{p}\left(1 \wedge \frac{d(y)}{|x-y|}\right)^{q} .
$$

If $[x y] \leq \frac{1}{2}[X Y]$ then

$$
\left(1 \wedge \frac{d(x)^{p} d(y)^{q}}{|x-y|^{p+q}}\right) \sim 1 \sim\left(1 \wedge \frac{d(x)}{|x-y|}\right)^{p}\left(1 \wedge \frac{d(y)}{|x-y|}\right)^{q} .
$$

ix. Lemma 3.1 implies if $[x y] \geq \frac{1}{2}[X Y]$ that

$$
\begin{gathered}
\log \left(1+\frac{d(x)^{p} d(y)^{q}}{|x-y|^{p+q}}\right) \sim \frac{d(x)^{p} d(y)^{q}}{|x-y|^{p+q}} \sim\left(1 \wedge \frac{d(x)^{p} d(y)^{q}}{|x-y|^{p+q}}\right) \sim \\
\sim \log \left(2+\frac{d(y)}{|x-y|}\right)\left(1 \wedge \frac{d(x)^{p} d(y)^{q}}{|x-y|^{p+q}}\right) .
\end{gathered}
$$

If $[x y] \leq \frac{1}{2}[X Y]$ then by Lemma 3.1

$$
\log \left(1+\frac{d(x)^{p} d(y)^{q}}{|x-y|^{p+q}}\right) \sim \log \left(1+\frac{d(y)^{p+q}}{|x-y|^{p+q}}\right) \sim
$$




$$
\begin{gathered}
\sim \log \left(\left(2+\frac{d(y)}{|x-y|}\right)^{p+q}\right) \sim \log \left(2+\frac{d(y)}{|x-y|}\right) \sim \\
\sim \log \left(2+\frac{d(y)}{|x-y|}\right)\left(1 \wedge \frac{d(x)^{p} d(y)^{q}}{|x-y|^{p+q}}\right) .
\end{gathered}
$$

Lemma 3.3 Let $r, s, t \geq 0$.

$i$. If $s+t \geq 2 r$, then on $B^{2}$ we have

$$
\left(1 \wedge \frac{d(x)^{t} d(y)^{s}}{|x-y|^{t+s}}\right) \preceq\left(\frac{d(y)}{d(x)}\right)^{r-t}\left(1 \wedge \frac{d(x)^{r} d(y)^{r}}{|x-y|^{2 r}}\right) .
$$

ii. If $s+t=2 r$, then on $B^{2}$ we have

$$
\left(1 \wedge \frac{d(x)^{t} d(y)^{s}}{|x-y|^{t+s}}\right) \sim\left(\frac{d(y)}{d(x)}\right)^{r-t}\left(1 \wedge \frac{d(x)^{r} d(y)^{r}}{|x-y|^{2 r}}\right) .
$$

By symmetry one may interchange $x, t$ with $y, s$.

Proof of Lemma 3.3. We will use Lemma 3.1 several times.

- The case $[x y] \leq \frac{1}{2}[X Y]$. We have

$$
\begin{gathered}
\left(1 \wedge \frac{d(x)^{t} d(y)^{s}}{|x-y|^{t+s}}\right) \sim 1 \sim\left(\frac{d(y)}{d(x)}\right)^{r-t} \sim \\
\sim\left(\frac{d(y)}{d(x)}\right)^{r-t}\left(1 \wedge \frac{d(x)^{r} d(y)^{r}}{|x-y|^{2 r}}\right) .
\end{gathered}
$$

- The case $[x y] \geq \frac{1}{2}[X Y]$. We have

$$
\begin{gathered}
\left(1 \wedge \frac{d(x)^{t} d(y)^{s}}{|x-y|^{t+s}}\right) \sim \frac{d(x)^{t} d(y)^{s}}{|x-y|^{t+s}}= \\
=\left(\frac{d(y)}{d(x)}\right)^{r-t} \frac{d(x)^{r} d(y)^{r}}{|x-y|^{2 r}} \frac{d(y)^{s+t-2 r}}{|x-y|^{s+t-2 r}} \preceq \\
\preceq\left(\frac{d(y)}{d(x)}\right)^{r-t} \frac{d(x)^{r} d(y)^{r}}{|x-y|^{2 r}} \sim \\
\sim\left(\frac{d(y)}{d(x)}\right)^{r-t}\left(1 \wedge \frac{d(x)^{r} d(y)^{r}}{|x-y|^{2 r}}\right) .
\end{gathered}
$$

Note that when $s+t=2 r$ we may replace $\preceq$ by $=$ in the previous estimate.

Before proving both propositions of the previous section we will introduce two convenient transformations of (5). For the case that $[x y] \geq \frac{1}{2}[X Y]$ we will use the 
integral transformation with $s=1-v^{-2}$ and we obtain the following expression for $G_{m, n}$ :

$$
G_{m, n}(x, y)=\frac{1}{2} k_{m, n}|x-y|^{2 m-n} \int_{s=0}^{A_{x y}} s^{m-1}(1-s)^{\frac{1}{2} n-m-1} d s
$$

where

$$
A_{x y}=\frac{[X Y]^{2}-[x y]^{2}}{[X Y]^{2}} \text {. }
$$

We set

$$
f_{m, n}(t)=\int_{s=0}^{t} s^{m-1}(1-s)^{\frac{1}{2} n-m-1} d s
$$

By straightforward calculus it follows that for $t \in[0,1]$, if $m \geq j>0$,

$$
\begin{gathered}
\left|f_{m, n}^{(j)}(t)\right| \preceq t^{m-j}(1-t)^{\frac{1}{2} n-m-j}\left(t^{j-1}+(1-t)^{j-1}\right) \preceq \\
\preceq t^{m-j}(1-t)^{\frac{1}{2} n-m-j}
\end{gathered}
$$

and if $m<j$

$$
\left|f_{m, n}^{(j)}(t)\right| \preceq(1-t)^{\frac{1}{2} n-m-j}\left(t^{m-1}+(1-t)^{m-1}\right) \preceq(1-t)^{\frac{1}{2} n-m-j} .
$$

For the case that $[x y] \leq \frac{1}{2}[X Y]$ we use the transformation with $w=v^{-1}$ and (5) becomes:

$$
G_{m, n}(x, y)=k_{m, n}|x-y|^{2 m-n} \int_{w=[x y] /[X Y]}^{1} w^{n-2 m-1}\left(1-w^{2}\right)^{m-1} d w .
$$

We also set

$$
g_{m, n}(t)=\int_{w=t}^{1} w^{n-2 m-1}\left(1-w^{2}\right)^{m-1} d w
$$

Proof of Proposition 2.3.

First we prove Proposition 2.3 i. which follows rather directly from (15). Indeed, if $n>2 m$, then one finds by distinguishing the cases $0 \leq t \leq \frac{1}{2}$ and $\frac{1}{2} \leq t \leq 1$ that $f_{m, n}$ in (17) satisfies

$$
f_{m, n}(t) \sim t^{m} \text { on }[0,1]
$$

and hence by (15), using respectively Lemma 3.2 iii./iv., i. and vi., that

$$
\begin{gathered}
G_{m, n}(x, y) \sim|x-y|^{2 m-n}\left(A_{x y}\right)^{m} \sim \\
\sim|x-y|^{2 m-n}\left(\frac{d(x) d(y)}{d(x)^{2}+d(y)^{2}+|x-y|^{2}}\right)^{m} \sim \\
\sim|x-y|^{2 m-n}\left(\min \left(\frac{d(y)}{d(x)}, \frac{d(x)}{d(y)}, \frac{d(x) d(y)}{|x-y|^{2}}\right)\right)^{m} \sim \\
\sim|x-y|^{2 m-n} \min \left(1,\left(\frac{d(x) d(y)}{|x-y|^{2}}\right)^{m}\right) .
\end{gathered}
$$


For Proposition 2.3 ii. and iii. we have to distinguish two cases.

- The case $[x y] \geq \frac{1}{2}[X Y]$. Then $A_{x y} \leq \frac{3}{4}$ and the formula in (15) implies

$$
\begin{gathered}
G_{m, n}(x, y) \sim|x-y|^{2 m-n}\left(A_{x y}\right)^{m} \sim \\
\sim|x-y|^{2 m-n} \frac{d(x)^{m} d(y)^{m}}{[X Y]^{2 m}} \sim \\
\sim \frac{d(x)^{m} d(y)^{m}}{[X Y]^{n}} \sim \\
\sim \frac{d(x)^{m} d(y)^{m}}{(d(x)+d(y)+|x-y|)^{n}} \sim \\
\sim d(x)^{m-\frac{1}{2} n} d(y)^{m-\frac{1}{2} n}\left(\min \left(\frac{d(x)}{d(y)}, \frac{d(y)}{d(x)}, \frac{d(x) d(y)}{|x-y|^{2}}\right)\right)^{\frac{1}{2} n} \sim \\
\sim d(x)^{m-\frac{1}{2} n} d(y)^{m-\frac{1}{2} n} \min \left(1,\left(\frac{d(x) d(y)}{|x-y|^{2}}\right)^{\frac{1}{2} n}\right) .
\end{gathered}
$$

In the last three steps we used respectively Lemma 3.2 iv., i. and vi. Notice that, if $2 m=n,(9)$ implies

$$
\begin{gathered}
d(x)^{m-\frac{1}{2} n} d(y)^{m-\frac{1}{2} n} \min \left(1,\left(\frac{d(x) d(y)}{|x-y|^{2}}\right)^{\frac{1}{2} n}\right) \sim \\
\sim \log \left(1+\left(\frac{d(x) d(y)}{|x-y|^{2}}\right)^{\frac{1}{2} n}\right) .
\end{gathered}
$$

- The case $[x y] \leq \frac{1}{2}[X Y]$. Now it follows from (20) that we find

$$
\begin{gathered}
G_{m, n}(x, y) \sim|x-y|^{2 m-n} \int_{w=[x y] /[X Y]}^{1} w^{n-2 m-1} d w \sim \\
\sim\left\{\begin{array}{cc}
\text { if } n=2 m: & \log ([X Y] /[x y]) ; \\
\text { if } n<2 m: & {[X Y]^{2 m-n} .}
\end{array}\right.
\end{gathered}
$$

For $n=2 m$ one uses $\log (1+a) \sim \log \left(1+a^{\frac{1}{2} n}\right)$ for $a \in\left[\frac{3}{4}, \infty\right)$, and continues by

$$
\begin{gathered}
\log \left(\frac{[X Y]}{[x y]}\right) \sim \log \left(1+\frac{[X Y]^{2}-[x y]^{2}}{[x y]^{2}}\right) \sim \\
\sim \log \left(1+\frac{d(x) d(y)}{|x-y|^{2}}\right) \sim \log \left(1+\left(\frac{d(x) d(y)}{|x-y|^{2}}\right)^{\frac{1}{2} n}\right) .
\end{gathered}
$$

In the last step we used (11). For $n<2 m$ one has, using respectively Lemma 3.2 iv., (11) and (12), and again (11)

$$
\begin{aligned}
{[X Y]^{2 m-n} } & \sim(d(x)+d(y)+|x-y|)^{2 m-n} \sim \\
& \sim(d(x) d(y))^{m-\frac{1}{2} n} \sim
\end{aligned}
$$




$$
\sim(d(x) d(y))^{m-\frac{1}{2} n} \min \left(1,\left(\frac{d(x) d(y)}{|x-y|^{2}}\right)^{\frac{1}{2} n}\right) .
$$

Proposition 2.4 will be a consequence of the following lemmas.

Lemma 3.4 We have on $\Omega_{1}:=\left\{(x, y) \in B^{2} ;[x y] \geq \frac{1}{2}[X Y]\right\}$ that

i. if $|k| \leq m$

$$
\left|D_{x}^{k} G_{m, n}(x, y)\right| \preceq|x-y|^{2 m-n-|k|}\left(\frac{d(x)}{[X Y]}\right)^{m-|k|}\left(\frac{d(y)}{[X Y]}\right)^{m} ;
$$

ii. if $|k| \geq m$

$$
\left|D_{x}^{k} G_{m, n}(x, y)\right| \preceq|x-y|^{2 m-n-|k|}\left(\frac{d(y)}{[X Y]}\right)^{m} .
$$

Proof of Lemma 3.4. We will use (15). With the assumption $[x y] \geq \frac{1}{2}[X Y]$ it is sufficient to estimate $f_{m, n}(t)$ on $\left[0, \frac{3}{4}\right]$ since $0 \leq A_{x y} \leq \frac{3}{4}$ for all $(x, y) \in \Omega_{1}$. We find by (17-19) that

$$
\left|f_{m, n}^{(j)}(t)\right| \preceq t^{\max (m-j, 0)} \quad \text { on }\left[0, \frac{3}{4}\right] \text { for all } j \geq 0 .
$$

Since

$$
\begin{gathered}
A_{x y}=\frac{\left(1-|x|^{2}\right)\left(1-|y|^{2}\right)}{[X Y]^{2}}, \\
\left|D_{x}^{p}[X Y]\right| \preceq[X Y]^{1-|p|},
\end{gathered}
$$

and $1-|x|^{2} \leq 2 d(x) \preceq[X Y]$ by Lemma 3.2 iv., it follows that on $B^{2}$

$$
\left|D_{x}^{p} A_{x y}\right| \preceq \frac{d(y)}{[X Y]^{|p|+1}} .
$$

By a tedious application of the chain rule we find that

$$
\begin{gathered}
\left|D_{x}^{k} G_{m, n}(x, y)\right| \preceq \\
\preceq \sum_{p \leq k}\left|D_{x}^{k-p}\right| x-\left.y\right|^{2 m-n}|| D_{x}^{p} f_{m, n}\left(A_{x y}\right) \mid \preceq \\
\preceq \sum_{p \leq k}\left|D_{x}^{k-p}\right| x-\left.y\right|^{2 m-n}\left|\sum_{j=0}^{|p|}\right| f_{m, n}^{(j)}\left(A_{x y}\right)\left|\sum_{\substack{\sum_{i=1}^{j} p^{(i)}=p \\
1 \leq p^{(i)} \mid}} \prod_{i=1}^{j}\right| D_{x}^{p^{(i)}} A_{x y} \mid=
\end{gathered}
$$

where the last sum is 1 for $j=0$. Note that (23) implies

$$
\sum_{\substack{\sum_{i=1}^{j} p^{(i)}=p \\ 1 \leq\left|p^{(i)}\right|}} \prod_{i=1}^{j}\left|D_{x}^{p^{(i)}} A_{x y}\right| \preceq \frac{d(y)^{j}}{[X Y]^{|p|+j}} .
$$


Using respectively (22), Lemma 3.2 iii. and iv., and again iv., we continue by

$$
\begin{gathered}
(24) \preceq \sum_{p \leq k}|x-y|^{2 m-n-|k|+|p|} \sum_{j=0}^{|p|}\left(A_{x y}\right)^{\max (m-j, 0)} \frac{d(y)^{j}}{[X Y]^{j+|p|}} \preceq \\
\preceq \sum_{p \leq k}|x-y|^{2 m-n-|k|+|p|} \sum_{j=0}^{|p|}\left(\frac{d(x) d(y)}{[X Y]^{2}}\right)^{\max (m-j, 0)} \frac{d(y)^{j}}{[X Y]^{j+|p|}} \preceq \\
\preceq \sum_{p \leq k}|x-y|^{2 m-n-|k|+|p|}\left(\frac{d(x)}{[X Y]}\right)^{\max (m-|p|, 0)}\left(\frac{d(y)}{[X Y]}\right)^{m}[X Y]^{-|p|} \preceq \\
\preceq|x-y|^{2 m-n-|k|}\left(\frac{d(x)}{[X Y]}\right)^{\max (m-|k|, 0)}\left(\frac{d(y)}{[X Y]}\right)^{m} .
\end{gathered}
$$

We used $\frac{d(x)}{[X Y]} \leq 1, \frac{d(y)}{[X Y]} \leq 1$ and $[x y] \sim[X Y]$.

Lemma 3.5 We have on $\Omega_{2}:=\left\{(x, y) \in B^{2} ;[x y] \leq \frac{1}{2}[X Y]\right\}$ that

i. if $|k|>2 m-n$

$$
\left|D_{x}^{k} G_{m, n}(x, y)\right| \preceq|x-y|^{2 m-n-|k|} ;
$$

ii. if $|k|=2 m-n$

$$
\begin{aligned}
& \left|D_{x}^{k} G_{m, n}(x, y)\right| \preceq \log \left(\frac{[X Y]}{[x y]}\right) \quad \text { for } n \text { even }, \\
& \left|D_{x}^{k} G_{m, n}(x, y)\right| \preceq 1 \quad \text { for } n \text { odd } ;
\end{aligned}
$$

iii. if $|k|<2 m-n$

$$
\left|D_{x}^{k} G_{m, n}(x, y)\right| \preceq[X Y]^{2 m-n-|k|} .
$$

Proof of Lemma 3.5. First we will derive some estimates for $g_{m, n}$ defined in (21). If $n>2 m$ then for all $j \geq 0$

$$
\left|g_{m, n}^{(j)}(t)\right| \preceq 1 \quad \text { on }[0,1] .
$$

If $n=2 m=2$ then

$$
g_{1,2}(t)=-\log (t)
$$

and if $n=2 m>2$ then

$$
g_{m, 2 m}(t)=-\log (t)+P_{2 m-2}(t),
$$

where $P_{2 m-2}$ is some polynomial of degree $2 m-2$. Hence if $n=2 m$ we get

$$
\left|g_{m, 2 m}(t)\right| \preceq-\log t \quad \text { on } \quad\left[0, \frac{1}{2}\right] .
$$


and for $j>0$ that

$$
\left|g_{m, 2 m}^{(j)}(t)\right| \preceq t^{-j} \quad \text { on } \quad\left[0, \frac{1}{2}\right] .
$$

Which implies that we obtain for $n \geq 2 m$ and $|p| \geq 1$ :

$$
\begin{gathered}
\left|D_{x}^{p} g_{m, n}\left(\frac{[x y]}{[X Y]}\right)\right| \preceq \\
\preceq \sum_{j=1}^{|p|}\left|g_{m, n}^{(j)}\left(\frac{[x y]}{[X Y]}\right)\right| \sum_{\substack{p^{(1)}+\ldots+p^{(j)}=p \\
\left|p^{(i)}\right| \geq 1}} \prod_{i=1}^{j}\left|D_{x}^{p^{(i)}}\left(\frac{[x y]}{[X Y]}\right)\right| \preceq \\
\preceq \sum_{j=1}^{|p|}\left(\frac{[x y]}{[X Y]}\right)^{-j} \sum_{\substack{p^{(1)}+\ldots+p^{(j)}=p \\
\left|p^{(i)}\right| \geq 1}} \prod_{i=1}^{j}\left(\frac{[x y]^{1-\left|p^{(i)}\right|}}{[X Y]}\right) \preceq \\
\left.\preceq\right|^{-j}-\left.y\right|^{-|p|} .
\end{gathered}
$$

Next we distinguish the cases $n>2 m, n=2 m$ and $n<2 m$.

- The case $n>2 m$. Inequality (28) implies that

$$
\begin{gathered}
\left|D_{x}^{k} G_{m, n}(x, y)\right| \preceq \\
\preceq \sum_{p \leq k}\left|D_{x}^{k-p}\right| x-\left.y\right|^{2 m-n}|| D_{x}^{p} g_{m, n}\left(\frac{[x y]}{[X Y]}\right) \mid \preceq \\
\preceq \sum_{p \leq k}|x-y|^{2 m-n-|k|+|p|}|x-y|^{-|p|} \preceq \\
\preceq|x-y|^{2 m-n-|k|} .
\end{gathered}
$$

- The case $n=2 m$. If $|k|=0$ the result follows from (25) and (26). If $|k| \geq 1$ we obtain by (28) that

$$
\left|D_{x}^{k} G_{m, n}(x, y)\right| \sim\left|D_{x}^{k} g_{m, n}\left(\frac{[x y]}{[X Y]}\right)\right| \preceq|x-y|^{-|k|} .
$$

- The case $n<2 m$. Integration by part yields

$$
\begin{gathered}
g_{m, n}(t)=\frac{1}{2 m-n} t^{n-2 m}\left(1-t^{2}\right)^{m-1}-\frac{2 m-2}{2 m-n} g_{m-1, n}(t)= \\
=\sum_{i=1}^{\ell} \frac{\alpha_{m, n, i}}{2(m-i)} t^{n-2 m+2 i-2}\left(1-t^{2}\right)^{m-i}-\alpha_{m, n, \ell} g_{m-\ell, n}(t)
\end{gathered}
$$

with

$$
\alpha_{m, n, i}=(-1)^{i-1} \frac{\left(\begin{array}{c}
m-1 \\
i
\end{array}\right)}{\left(\begin{array}{c}
m-\frac{1}{2} n \\
i
\end{array}\right)},
$$

for all $\ell \leq m-\frac{1}{2} n$ if $n$ is even and for all $\ell<m$ if $n$ is odd. Define

$$
H_{m, n, \ell}(x, y)=\sum_{i=1}^{\ell} \frac{\alpha_{m, n, i}}{2(m-i)}[x y]^{2 i-2}[X Y]^{2 m-n-2 i+2}\left(A_{x y}\right)^{m-i}
$$


and we have

$$
G_{m, n}(x, y)=H_{m, n, \ell}(x, y)-\alpha_{m, n, \ell}[x y]^{2 m-n} g_{m-\ell, n}\left(\frac{[x y]}{[X Y]}\right) .
$$

From

$$
\left|D_{x}^{k}[x y]^{2 i-2}\right|=0 \quad \text { for } k>2 i-2,
$$

since $2 i-2$ is even and positive, and (23), we get for $\ell<m$ that

$$
\begin{gathered}
\left|D_{x}^{k} H_{m, n, \ell}(x, y)\right| \preceq \\
\preceq \sum_{i=1}^{\ell} \sum_{p \leq k}\left|D_{x}^{k-p}\right| x-\left.y\right|^{2 i-2}|| D_{x}^{p}\left([X Y]^{2 m-n-2 i+2}\left(A_{x y}\right)^{m-i}\right) \mid \preceq \\
\preceq \sum_{i=1}^{\ell} \sum_{\substack{p \leq k \\
|k|+2-2 i \leq|p|}}|x-y|^{2 i-2-|k|+|p|}[X Y]^{2 m-n-2 i+2-|p|} \preceq \\
\preceq[X Y]^{2 m-n-|k|}
\end{gathered}
$$

Hence $H_{m, n, \ell}(x, y)$ for $\ell<m$ is the innocent part. The estimate for the derivatives of $[x y]^{2 m-n} g_{m-\ell, n}([x y] /[X Y])$ will be crucial.

We distinguish further the cases $n$ is even and $n$ odd.

-• $n$ even. We use (29) with $\ell=m-\frac{1}{2} n$ and we get that

$$
\begin{gathered}
\left|D_{x}^{k}\left([x y]^{2 m-n} g_{\frac{1}{2} n, n}\left(\frac{[x y]}{[X Y]}\right)\right)\right| \preceq \\
\preceq \sum_{\substack{p \leq k \\
|k|-2 m+n \leq|p|}}\left|D_{x}^{k-p}\right| x-\left.y\right|^{2 m-n}|| D_{x}^{p} g_{\frac{1}{2} n, n}\left(\frac{[x y]}{[X Y]}\right) \mid=
\end{gathered}
$$

A next distinction will be $|k| \leq 2 m-n$ and $|k|>2 m-n$.

$\bullet \bullet n$ even and $|k| \leq 2 m-n$. Using (28) and (26) we get

$$
\begin{gathered}
(31) \preceq|x-y|^{2 m-n-|k|} g_{\frac{1}{2} n, n}\left(\frac{[x y]}{[X Y]}\right)+\sum_{\substack{p \leq k \\
1 \leq|p|}}|x-y|^{2 m-n-|k|+|p|}|x-y|^{-|p|} \preceq \\
\preceq|x-y|^{2 m-n-|k|} \log \left(\frac{[X Y]}{[x y]}\right)+|x-y|^{2 m-n-|k|} \preceq \\
\preceq|x-y|^{2 m-n-|k|} \log \left(\frac{[X Y]}{[x y]}\right) .
\end{gathered}
$$

With (30) we find if $|k|=2 m-n$ that

$$
\left|D_{x}^{k} G_{m, n}(x, y)\right| \preceq \log \left(\frac{[X Y]}{[x y]}\right),
$$

and if $|k|<2 m-n$ then

$$
|x-y|^{2 m-n-|k|} \log \left(\frac{[X Y]}{[x y]}\right) \preceq[X Y]^{2 m-n-|k|}
$$


which implies

$$
\left|D_{x}^{k} G_{m, n}(x, y)\right| \preceq[X Y]^{2 m-n-|k|} .
$$

$\bullet \bullet n$ even and $|k|>2 m-n$. With (28) we get

$$
\begin{gathered}
(31) \preceq \sum_{\substack{p \leq k \\
|k|-2 m+n \leq|p|}}|x-y|^{2 m-n-|k|+|p|}|x-y|^{-|p|} \preceq \\
\preceq|x-y|^{2 m-n-|k|} .
\end{gathered}
$$

-• $n$ odd. If $n=1$ we proceed straightforwardly from Boggio's formula:

$$
\begin{gathered}
G_{m, 1}(x, y)= \\
=c_{m, 1}|x-y|^{2 m-1} \int_{v=1}^{[X Y] /[x y]}\left(v^{2}-1\right)^{m-1} d v= \\
=\sum_{q=0}^{m-1} \alpha_{q}[X Y]^{2 m-2 q-1}[x y]^{2 q}+\alpha_{m}[x y]^{2 m-1} .
\end{gathered}
$$

Hence it follows that

$$
\left|D_{x}^{k} G_{m, n}(x, y)\right| \preceq
$$

$$
\begin{gathered}
\preceq \sum_{q=0}^{m-1} \sum_{p \leq k}\left|D_{x}^{k-p}[X Y]^{2 m-2 q-1}\right|\left|D_{x}^{p}[x y]^{2 q}\right|+\left|D_{x}^{k}[x y]^{2 m-1}\right| \preceq \\
\preceq \sum_{q=0}^{m-1} \sum_{\substack{p \leq k \\
|p| \leq 2 q}}[X Y]^{2 m-2 q-1-|k|+|p|}[x y]^{2 q-|p|}+[x y]^{2 m-1-|k|} \preceq \\
\preceq[X Y]^{2 m-1-|k|}+[x y]^{2 m-1-|k|} .
\end{gathered}
$$

If $|k| \leq 2 m-1$, then $[X Y]^{2 m-1-|k|}+[x y]^{2 m-1-|k|} \preceq[X Y]^{2 m-1-|k|}$ and if $|k|>2 m-1$, then $[X Y]^{2 m-1-|k|}+[x y]^{2 m-1-|k|} \preceq[x y]^{2 m-1-|k|}$.

If $n>1$ is odd we use (29) with $\ell=m-\frac{1}{2}(n-1)$ to find

$$
\begin{gathered}
G_{m, n}(x, y)= \\
=H_{m, n, m-\frac{1}{2}(n-1)}(x, y)-\alpha_{m, n, m-\frac{1}{2}(n-1)}[x y]^{2 m-n} g_{\frac{1}{2} n-\frac{1}{2}, n}\left(\frac{[x y]}{[X Y]}\right) .
\end{gathered}
$$

Notice that, since $n-3$ is nonnegative and even,

$$
g_{\frac{1}{2} n-\frac{1}{2}, n}(t)=\int_{t}^{1}\left(1-w^{2}\right)^{\frac{n-3}{2}} d w
$$

is a polynomial in $[x y] /[X Y]$. We find that

$$
\left|D_{x}^{k}\left([x y]^{2 m-n} g_{\frac{1}{2} n-\frac{1}{2}, n}\left(\frac{[x y]}{[X Y]}\right)\right)\right| \preceq|x-y|^{2 m-n-|k|} .
$$


Hence, combining (30) and (32) we find that

$$
\left|D_{x}^{k} G_{m, n}(x, y)\right| \preceq[X Y]^{2 m-n-|k|}+|x-y|^{2 m-n-|k|},
$$

which implies if $|k| \geq 2 m-n$ that

$$
\left|D_{x}^{k} G_{m, n}(x, y)\right| \preceq|x-y|^{2 m-n-|k|},
$$

and if $|k| \leq 2 m-n$ that

$$
\left|D_{x}^{k} G_{m, n}(x, y)\right| \preceq[X Y]^{2 m-n-|k|} .
$$

Proof of Proposition 2.4. Again we separate the two cases.

- The case $[x y] \geq \frac{1}{2}[X Y]$. We find by (10) that on $\Omega_{1}$ :

$$
\frac{d(x)}{[X Y]} \sim \frac{d(x)}{[x y]} \preceq 1 \text { and } \frac{d(y)}{[X Y]} \sim \frac{d(y)}{[x y]} \preceq 1
$$

and

$$
\log \left(2+\frac{d(y)}{|x-y|}\right) \sim 1 .
$$

Since $t \sim(1 \wedge t)$ for $t \in[0, M]$ and $M$ fixed, we find that Lemma 3.4 i. implies Proposition 2.4 i./ii. (a) and that Lemma 3.4 ii. implies Proposition 2.4 i./ii. (b). It remains to prove Proposition 2.4 iii.

If $m-\frac{1}{2} n \geq|k|$ it follows from Lemma $3.4 \mathrm{i}$. and (33) that

$$
\begin{gathered}
\left|D_{x}^{k} G_{m, n}(x, y)\right| \preceq|x-y|^{2 m-n-|k|} \frac{d(x)^{m-|k|}}{|x-y|^{m-|k|}} \frac{d(y)^{m}}{|x-y|^{m}}= \\
=d(x)^{m-\frac{1}{2} n-|k|} d(y)^{m-\frac{1}{2} n} \frac{d(x)^{\frac{1}{2} n} d(y)^{\frac{1}{2} n}}{|x-y|^{n}} \preceq \\
\preceq d(x)^{m-\frac{1}{2} n-|k|} d(y)^{m-\frac{1}{2} n}\left(1 \wedge \frac{d(x)^{\frac{1}{2} n} d(y)^{\frac{1}{2} n}}{|x-y|^{n}}\right) .
\end{gathered}
$$

which implies Proposition 2.4 iii.(a).

If $m \geq|k| \geq m-\frac{1}{2} n$ it follows from Lemma 3.4 i. and (33) that

$$
\begin{gathered}
\left|D_{x}^{k} G_{m, n}(x, y)\right| \preceq|x-y|^{2 m-n-|k|} \frac{d(x)^{m-|k|}}{|x-y|^{m-|k|}} \frac{d(y)^{m}}{|x-y|^{m}}= \\
=d(y)^{2 m-n-|k|} \frac{d(x)^{m-|k|} d(y)^{n-m+|k|}}{|x-y|^{n}} \preceq \\
\preceq d(y)^{2 m-n-|k|}\left(1 \wedge \frac{d(x)^{m-|k|} d(y)^{n-m+|k|}}{|x-y|^{n}}\right)
\end{gathered}
$$

which implies Proposition 2.4 iii.(b).

If $2 m-n \geq|k| \geq m$ it follows from Lemma 3.4 ii. and (33) that

$$
\left|D_{x}^{k} G_{m, n}(x, y)\right| \preceq|x-y|^{2 m-n-|k|} \frac{d(y)^{m}}{|x-y|^{m}}=
$$




$$
=d(y)^{2 m-n-|k|} \frac{d(y)^{n-m+|k|}}{|x-y|^{n-m+|k|}} \preceq d(y)^{2 m-n-|k|}\left(1 \wedge \frac{d(y)^{n-m+|k|}}{|x-y|^{n-m+|k|}}\right),
$$

which implies Proposition 2.4 iii.(c).

- The case $[x y] \leq \frac{1}{2}[X Y]$. From (11-12) we find that on $\Omega_{2}$

$$
\frac{d(x)}{|x-y|} \succeq 1 \text { and } \frac{d(y)}{|x-y|} \succeq 1 .
$$

Hence Lemma 3.5 i. implies Proposition 2.4 i. (a) and (b). Since (11) shows that on $\Omega_{2}$

$$
[X Y] \preceq d(x) \text { and }[X Y] \preceq d(y) \text {, }
$$

Lemma 3.5 iii. implies Proposition 2.4 iii. (a), (b) and (c). For Proposition 2.4 ii. notice that on $\Omega_{2}$

$$
\begin{gathered}
\log \left(\frac{[X Y]}{[x y]}\right) \preceq \log \left(2+\frac{d(y)}{|x-y|}\right) \sim \\
\sim \log \left(2+\frac{d(y)}{|x-y|}\right)\left(1 \wedge \frac{d(x)^{(m-|k|)^{+}} d(y)^{m}}{|x-y|^{(m-|k|)^{+}+m}}\right),
\end{gathered}
$$

implying both (a) and (b).

\section{Results of 3-G type}

Proposition 4.1 For $k \in \mathbb{N}^{n}$ with $|k| \leq 2 m$ the following holds on $B^{3}(x, y, z \in B)$.

i. If $2 m \geq|k|>2 m-n$, then:

$$
\frac{G_{m, n}(x, z)\left|D_{z}^{k} G_{m, n}(z, y)\right|}{G_{m, n}(x, y)} \preceq|x-z|^{2 m-n-|k|}+|z-y|^{2 m-n-|k|} ;
$$

ii. if $|k|=2 m-n$ with $n$ even, then

$$
\frac{G_{m, n}(x, z)\left|D_{z}^{k} G_{m, n}(z, y)\right|}{G_{m, n}(x, y)} \preceq \log \left(\frac{3}{|x-z|}\right)+\log \left(\frac{3}{|z-y|}\right) ;
$$

iii. if either $|k|=2 m-n$ with $n$ odd, or $|k|<2 m-n$, then:

$$
\frac{G_{m, n}(x, z)\left|D_{z}^{k} G_{m, n}(z, y)\right|}{G_{m, n}(x, y)} \preceq 1 .
$$

Corollary 4.2 There exists $M \in \mathbb{R}^{+}$, depending on $n, m$ only, such that for $k \in \mathbb{N}^{n}$ with $|k| \in[0,2 m-1]$ the following holds. Let $f \in L^{p}(B), p>1$, with $f \geq 0$ and we have

$$
\left|\left(\mathcal{G}_{m, n} D^{k} \mathcal{G}_{m, n} f\right)(x)\right| \leq M\left(\mathcal{G}_{m, n} f\right)(x) \quad \text { for almost all } x \in B \text {. }
$$


Proof of Corollary 4.2. Note that, due to the additional assumption $|k| \leq 2 m-1$, all right hand sides in Proposition 4.1 are uniformly integrable. That means there exists $M \in \mathbb{R}^{+}$such that for all $x, y \in B$

$$
\int_{z \in B} \frac{G_{m, n}(x, z)\left|D_{z}^{k} G_{m, n}(z, y)\right|}{G_{m, n}(x, y)} d z \leq M .
$$

Hence, since $|k| \leq 2 m-1$ by dominated convergence, see also [8, Lemma 4.1], and Fubini-Tonelli

$$
\begin{gathered}
\left|\left(\mathcal{G}_{m, n} D^{k} \mathcal{G}_{m, n} f\right)(x)\right|= \\
=\left|\int_{z \in B} G_{m, n}(x, z) D_{z}^{k} \int_{y \in B} G_{m, n}(z, y) f(y) d y d z\right| \leq \\
\leq \int_{y \in B} \int_{z \in B} G_{m, n}(x, z)\left|D_{z}^{k} G_{m, n}(z, y)\right| d z f(y) d y \leq \\
\leq M \int_{y \in B} G_{m, n}(x, y) f(y) d y=M\left(\mathcal{G}_{m, n} f\right)(x) .
\end{gathered}
$$

Before proving Proposition 4.1 we need a technical lemma.

Lemma 4.3 On $B^{3}(x, y, z \in B)$ one finds that

$$
\begin{aligned}
& \text { i. } T(x, y, z):=\frac{\left(1 \wedge \frac{d(x) d(z)}{|x-z|^{2}}\right)\left(1 \wedge \frac{d(z) d(y)}{|y-z|^{2}}\right)}{\left(1 \wedge \frac{d(x) d(y)}{|x-y|^{2}}\right)} \preceq 1 ; \\
& \text { ii. } Q(x, y, z):=\frac{\left(1 \wedge \frac{d(x) d(z)}{|x-z|^{2}}\right)\left(1 \wedge \frac{d(y)}{|y-z|}\right)}{\left(1 \wedge \frac{d(x) d(y)}{|x-y|^{2}}\right)} \preceq 1+\frac{|x-y|}{|x-z|} ; \\
& \text { iii. } L(x, y, z):=\frac{\log \left(1+\frac{d(x) d(z)}{|x-z|^{2}}\right)\left(1 \wedge \frac{d(y)}{|y-z|}\right)}{\log \left(1+\frac{d(x) d(y)}{|x-y|^{2}}\right)} \preceq 1+\frac{|x-y|}{|x-z|} ; \\
& \text { iv. } R(x, y, z):=\frac{|x-y|}{|x-z||z-y|} \leq \frac{1}{|x-z|}+\frac{1}{|z-y|} .
\end{aligned}
$$

Proof of Lemma 4.3. First we suppose that $[x y] \leq \frac{1}{2}[X Y]$. Hence Lemma 3.1 implies $\frac{d(x) d(y)}{|x-y|^{2}} \succeq \frac{d(x)}{|x-y|} \succeq 1$ and the estimates in i. and ii. follow from $\left(1 \wedge \frac{d(x) d(y)}{|x-y|^{2}}\right) \sim 1$. The estimate in iii. uses

$$
\frac{\log (1+t)}{\log (1+s)} \leq 1+\frac{t}{s} \quad \text { for } t, s \in \mathbb{R}^{+}
$$

Indeed, let $s \geq 0$ and one finds if $\alpha \geq 1$ that $\log (1+\alpha s) \leq \alpha \log (1+s)$ by the concavity of $\log$; if $\alpha \in[0,1]$ one finds $\log (1+\alpha s) \leq \log (1+s)$; (34) follows with $\alpha s=t$. From Lemma 3.2 ix. we find

$$
\log \left(1+\frac{d(x) d(y)}{|x-y|^{2}}\right) \sim \log \left(2+\frac{d(x)}{|x-y|}\right) \sim \log \left(1+\frac{d(x)}{|x-y|}\right) \succeq 1
$$


and

$$
\log \left(1+\frac{d(x) d(z)}{|x-z|^{2}}\right) \preceq \log \left(2+\frac{d(x)}{|x-z|}\right) \preceq 1+\log \left(1+\frac{d(x)}{|x-z|}\right) .
$$

Hence it follows that

$$
L(x, y, z) \preceq \frac{1+\log \left(1+\frac{d(x)}{|x-z|}\right)}{\log \left(1+\frac{d(x)}{|x-y|}\right)} \preceq 1+\frac{\log \left(1+\frac{d(x)}{|x-z|}\right)}{\log \left(1+\frac{d(x)}{|x-y|}\right)} \preceq 1+\frac{|x-y|}{|x-z|} .
$$

If $[x y] \geq \frac{1}{2}[X Y]$, then

$$
\left(1 \wedge \frac{d(x) d(y)}{|x-y|^{2}}\right) \sim \log \left(1+\frac{d(x) d(y)}{|x-y|^{2}}\right) \sim \frac{d(x) d(y)}{|x-y|^{2}}
$$

and we distinguish the following cases.

- The case $|x-z| \geq \frac{1}{2}|x-y|$. Using Lemma 3.2 vi. we get

$$
T(x, y, z) \preceq \frac{|x-y|^{2}}{d(x) d(y)} \frac{d(x) d(z)}{|x-z|^{2}} \frac{d(y)}{d(z)} \leq 4,
$$

and using Lemma $3.2 \mathrm{v}$. we obtain

$$
Q(x, y, z) \preceq \frac{|x-y|^{2}}{d(x) d(y)} \frac{d(x) d(z)}{|x-z|^{2}} \frac{d(y)}{d(z)} \leq 4 .
$$

Similarly we obtain that

$$
L(x, y, z) \preceq \frac{|x-y|^{2}}{d(x) d(y)} \frac{d(x) d(z)}{|x-z|^{2}} \frac{d(y)}{d(z)} \leq 4 .
$$

- The case $|x-z| \leq \frac{1}{2}|x-y|$. Then $|y-z| \geq|y-x|-|x-z| \geq \frac{1}{2}|x-y|$ and one proceeds for i. as in (35) using $T(x, y, z)=T(y, x, z)$. In order to prove ii. we proceed by using Lemma 3.2 vii.:

$$
Q(x, y, z) \preceq \frac{|x-y|^{2}}{d(x) d(y)} \frac{d(x)}{|x-z|} \frac{d(y)}{|y-z|} \leq 2 \frac{|x-y|}{|x-z|} .
$$

Similarly we find by Lemma 3.2 ix.,viii. and again ix.:

$$
L(x, y, z) \preceq \frac{|x-y|^{2}}{d(x) d(y)} \log \left(1+\frac{d(x)}{|x-z|}\right) \frac{d(y)}{|y-z|} \leq 2 \frac{|x-y|}{|x-z|} .
$$

Note that the estimate in iv. is straightforward.

Proof of Proposition 4.1.

- The case $n>2 m$. One finds by Proposition 2.3, Proposition 2.4 i., respectively,

(6) and Lemma 4.3 that

$$
\frac{G_{m, n}(x, z)\left|D_{z}^{k} G_{m, n}(z, y)\right|}{G_{m, n}(x, y)} \preceq
$$




$$
\begin{gathered}
\preceq \frac{|x-y|^{n-2 m}\left(1 \wedge \frac{d(x)^{m} d(z)^{m}}{|x-z|^{2 m}}\right)\left(1 \wedge \frac{d(z)^{(m-|k|)^{+}} d(y)^{m}}{|y-z|^{(m-|k|)^{+}+m}}\right)}{\left(1 \wedge \frac{d(x)^{m} d(y)^{m}}{|x-y|^{2 m}}\right)|x-z|^{n-2 m}|z-y|^{n-2 m+|k|}}= \\
=\frac{|x-y|^{n-2 m}}{|x-z|^{n-2 m}|z-y|^{n-2 m+|k|}}(T(x, y, z))^{(m-|k|)^{+}}(Q(x, y, z))^{m \wedge|k|} \preceq \\
\preceq \frac{|x-y|^{n-2 m}}{|x-z|^{n-2 m}|z-y|^{n-2 m+|k|}}\left(1+\left(\frac{|x-y|}{|x-z|}\right)^{m \wedge|k|}\right)= \\
=\frac{1}{|z-y|^{|k|}} R(x, y, z)^{n-2 m}+\frac{1}{|z-y|^{(|k|-m)^{+}} R(x, y, z)^{n-2 m+(m \wedge|k|)} \preceq} \\
\preceq \frac{1}{|z-y|^{|k|}|x-z|^{n-2 m}}+\frac{1}{|z-y|^{|k|+n-2 m}+} \\
+\frac{1}{|z-y|^{(|k|-m)^{+}}|x-z|^{n-2 m+(m \wedge|k|)} \preceq} \\
\preceq|x-z|^{2 m-n-|k|}+|z-y|^{2 m-n-|k|} .
\end{gathered}
$$

- $n=2 m$ and $0<|k| \leq 2 m$. By using Lemma 3.2 ix. we have

$$
\begin{gathered}
\frac{G_{m, n}(x, z)\left|D_{z}^{k} G_{m, n}(z, y)\right|}{G_{m, n}(x, y)} \preceq \\
\preceq \frac{\log \left(1+\frac{d(x) d(z)}{|x-z|^{2}}\right)\left(1 \wedge \frac{d(x)^{m-1} d(z)^{m-1}}{|x-z|^{2 m-2}}\right)}{\log \left(1+\frac{d(x) d(y)}{|x-y|^{2}}\right)\left(1 \wedge \frac{d(x)^{m-1} d(y)^{m-1}}{|x-y|^{2 m-2}}\right)} . \\
\cdot|y-z|^{2 m-n-|k|}\left(1 \wedge \frac{d(z)^{(m-|k|)^{+}} d(y)^{m}}{|y-z|^{(m-|k|)^{+}+m}}\right)= \\
=\frac{|x-y|^{n-2 m}}{|x-z|^{n-2 m}|z-y|^{n-2 m+|k|}} \cdot \\
\cdot(T(x, y, z))^{(m-|k|)^{+}} L(x, y, z)(Q(x, y, z))^{(m \wedge|k|)-1} .
\end{gathered}
$$

Since $L$ and $Q$ can be bounded similarly (see Lemma 4.3 ii. and iii.) we may continue as in (38).

- $n=2 m$ and $|k|=0$. We find by using Lemma 3.2 ix., the estimate for $T(x, y, z)$ and the symmetry of $G_{m, n}(x, y)$ that

$$
\begin{gathered}
\frac{G_{m, n}(x, z) G_{m, n}(z, y)}{G_{m, n}(x, y)} \preceq \\
\preceq \frac{\log \left(2+\frac{d(x)}{|x-z|}\right)\left(1 \wedge \frac{d(x)^{m} d(z)^{m}}{|x-z|^{2 m}}\right) \log \left(2+\frac{d(y)}{|y-z|}\right)\left(1 \wedge \frac{d(z)^{m} d(y)^{m}}{|y-z|^{2 m}}\right)}{\max \left(\log \left(2+\frac{d(x)}{|x-y|}\right), \log \left(2+\frac{d(y)}{|x-y|}\right)\right)\left(1 \wedge \frac{d(x)^{m} d(y)^{m}}{|x-y|^{2 m}}\right)} \preceq
\end{gathered}
$$




$$
\preceq \frac{\log \left(2+\frac{d(x)}{|x-z|}\right) \log \left(2+\frac{d(y)}{|y-z|}\right)}{\max \left(\log \left(2+\frac{d(x)}{|x-y|}\right), \log \left(2+\frac{d(y)}{|x-y|}\right)\right)}=
$$

If $|x-z| \geq \frac{1}{2}|x-y|$ then $\log \left(2+\frac{d(x)}{|x-z|}\right) \preceq \log \left(2+\frac{d(x)}{|x-y|}\right)$ and we continue by

$$
(39) \preceq \log \left(2+\frac{d(y)}{|y-z|}\right) \preceq \log \left(\frac{3}{|y-z|}\right) .
$$

If $|x-z| \leq \frac{1}{2}|x-y|$ then $|y-z| \geq|y-x|-|x-z| \geq \frac{1}{2}|x-y|$ and we conclude by

$$
(39) \preceq \log \left(2+\frac{d(x)}{|x-z|}\right) \preceq \log \left(\frac{3}{|x-z|}\right) .
$$

- $n<2 m$ and $0 \leq|k|<2 m-n$, or, $n<2 m$ and $|k|=2 m-n$ and $n$ odd. Note that by Lemma 3.3 (see also (8)) we may estimate as follows:

$$
\begin{gathered}
\frac{G_{m, n}(x, z)\left|D_{z}^{k} G_{m, n}(z, y)\right|}{G_{m, n}(x, y)} \preceq \\
\preceq \frac{(d(x) d(z))^{m-\frac{1}{2} n}\left(1 \wedge \frac{d(x) d(z)}{|x-z|^{2}}\right)^{\frac{1}{2} n}}{(d(x) d(y))^{m-\frac{1}{2} n}\left(1 \wedge \frac{d(x) d(y)}{|x-y|^{2}}\right)^{\frac{1}{2} n}} \\
\cdot d(z)^{m-\frac{1}{2} n-|k|} d(y)^{m-\frac{1}{2} n}\left(1 \wedge \frac{d(z) d(y)}{|y-z|^{2}}\right)^{\frac{1}{2} n}= \\
=d(z)^{2 m-n-|k|}(T(x, y, z))^{\frac{1}{2} n} \preceq 1
\end{gathered}
$$

- $n<2 m$ and $|k|=2 m-n$ with $n$ even. We use Lemma 3.3. Note that $|k|=2 m-n$ implies $m-|k|=n-m$. We have

$$
\begin{gathered}
\frac{G_{m, n}(x, z)\left|D_{z}^{k} G_{m, n}(z, y)\right|}{G_{m, n}(x, y)} \preceq \\
\preceq \frac{(d(x) d(z))^{m-\frac{1}{2} n}\left(1 \wedge \frac{d(x) d(z)}{|x-z|^{2}}\right)^{\frac{1}{2} n} \log \left(2+\frac{d(y)}{|z-y|}\right)\left(1 \wedge \frac{d(z)^{(m-|k|)^{+}} d(y)^{m}}{|y-z|^{(m-|k|)^{+}+m}}\right)}{(d(x) d(y))^{m-\frac{1}{2} n}\left(1 \wedge \frac{d(x) d(y)}{|x-y|^{2}}\right)^{\frac{1}{2} n}} \preceq \\
\preceq \frac{d(z)^{m-\frac{1}{2} n}\left(1 \wedge \frac{d(x) d(z)}{|x-z|^{2}}\right)^{\frac{1}{2} n} \log \left(2+\frac{d(y)}{|z-y|}\right)\left(\frac{d(y)}{d(z)}\right)^{m-\frac{1}{2} n}\left(1 \wedge \frac{d(z) d(y)}{|y-z|^{2}}\right)^{\frac{1}{2} n}}{d(y)^{m-\frac{1}{2} n}\left(1 \wedge \frac{d(x) d(y)}{|x-y|^{2}}\right)^{\frac{1}{2} n}}= \\
=\log \left(2+\frac{d(y)}{|z-y|}\right)(T(x, y, z))^{\frac{1}{2} n} \preceq \log \left(\frac{3}{|z-y|}\right) .
\end{gathered}
$$

- $n<2 m$ and $2 m-n<|k| \leq 2 m-\frac{1}{2} n$. We find that

$$
\frac{G_{m, n}(x, z)\left|D_{z}^{k} G_{m, n}(z, y)\right|}{G_{m, n}(x, y)} \preceq
$$




$$
\begin{aligned}
& \preceq \frac{(d(x) d(z))^{m-\frac{1}{2} n}\left(1 \wedge \frac{d(x) d(z)}{|x-z|^{2}}\right)^{\frac{1}{2} n}|z-y|^{2 m-n-|k|}\left(1 \wedge \frac{d(z)^{(m-|k|)^{+}} d(y)^{m}}{|y-z|^{(m-|k|)^{+}+m}}\right)}{(d(x) d(y))^{m-\frac{1}{2} n}\left(1 \wedge \frac{d(x)^{\frac{1}{2} n} d(y)^{\frac{1}{2} n}}{|x-y|^{n}}\right)}= \\
& =|z-y|^{2 m-n-|k|} \frac{\left(\frac{d(z)}{d(y)}\right)^{m-\frac{1}{2} n}\left(1 \wedge \frac{d(x) d(z)}{|x-z|^{2}}\right)^{\frac{1}{2} n}\left(1 \wedge \frac{d(z)^{(m-|k|)^{+}} d(y)^{m}}{|y-z|^{(m-|k|)^{+}+m}}\right)}{\left(1 \wedge \frac{d(x)^{\frac{1}{2} n} d(y)^{\frac{1}{2} n}}{|x-y|^{n}}\right)}=
\end{aligned}
$$

By Lemma 3.3 it follows that

$$
\left(1 \wedge \frac{d(y) d(z)}{|y-z|^{2}}\right)^{m-\frac{1}{2} n} \sim\left(1 \wedge \frac{d(z)}{|y-z|}\right)^{2 m-n}\left(\frac{d(y)}{d(z)}\right)^{m-\frac{1}{2} n} .
$$

By Lemma 3.2 and (41) it follows that

$$
\begin{gathered}
\left(1 \wedge \frac{d(z)^{(m-|k|)^{+}} d(y)^{m}}{|y-z|^{(m-|k|)^{+}+m}}\right) \sim \\
\sim\left(1 \wedge \frac{d(z) d(y)}{|y-z|^{2}}\right)^{2 m-\frac{1}{2} n-|k|}\left(1 \wedge \frac{d(y)}{|y-z|}\right)^{|k|+n-2 m} \cdot \\
\cdot\left(1 \wedge \frac{d(z)}{|y-z|}\right)^{(|k|-m)^{+}}\left(1 \wedge \frac{d(z)}{|y-z|}\right)^{n-2 m}\left(1 \wedge \frac{d(y) d(z)}{|y-z|^{2}}\right)^{m-\frac{1}{2} n} \preceq \\
\preceq\left(1 \wedge \frac{d(z) d(y)}{|y-z|^{2}}\right)^{2 m-\frac{1}{2} n-|k|}\left(1 \wedge \frac{d(y)}{|y-z|}\right)^{|k|+n-2 m}\left(\frac{d(y)}{d(z)}\right)^{m-\frac{1}{2} n},
\end{gathered}
$$

and using (42) we continue by

$$
\begin{gathered}
(40) \preceq|z-y|^{2 m-n-|k|} . \\
\cdot \frac{\left(1 \wedge \frac{d(x) d(z)}{|x-z|^{2}}\right)^{\frac{1}{2} n}\left(1 \wedge \frac{d(z) d(y)}{|y-z|^{2}}\right)^{2 m-\frac{1}{2} n-|k|}\left(1 \wedge \frac{d(y)}{|y-z|}\right)^{|k|+n-2 m}}{\left(1 \wedge \frac{d(x)^{\frac{1}{2} n} d(y)^{\frac{1}{2} n}}{|x-y|^{n}}\right)}= \\
=|z-y|^{2 m-n-|k|}(T(x, y, z))^{2 m-\frac{1}{2} n-|k|}(Q(x, y, z))^{|k|+n-2 m} \preceq \\
\preceq|z-y|^{2 m-n-|k|}\left(1+\frac{|x-y|}{|x-z|}\right)^{|k|+n-2 m} \preceq \\
\preceq|z-y|^{2 m-n-|k|}+|x-z|^{2 m-n-|k|} .
\end{gathered}
$$

- $n<2 m$ and $2 m-\frac{1}{2} n<|k| \leq 2 m$. Similar as in the previous case we obtain the estimate in (40). By Lemma 3.2 we get

$$
\left(1 \wedge \frac{d(z)^{(m-|k|)^{+}} d(y)^{m}}{|y-z|^{(m-|k|)^{+}+m}}\right) \preceq\left(\frac{d(y)}{d(z)}\right)^{m-\frac{1}{2} n}\left(1 \wedge \frac{d(y)}{|y-z|}\right)^{\frac{1}{2} n},
$$


and we continue by

$$
\begin{gathered}
(40) \preceq|y-z|^{2 m-n-|k|} \frac{\left(1 \wedge \frac{d(x) d(z)}{|x-z|^{2}}\right)^{\frac{1}{2} n}\left(1 \wedge \frac{d(y)}{|y-z|}\right)^{\frac{1}{2} n}}{\left(1 \wedge \frac{d(x)^{\frac{1}{2} n} d(y)^{\frac{1}{2} n}}{|x-y|^{n}}\right)}= \\
=|z-y|^{2 m-n-|k|}(Q(x, y, z))^{\frac{1}{2} n} \preceq \\
\preceq|z-y|^{2 m-n-|k|}\left(1+\left(\frac{|x-y|}{|x-z|}\right)^{\frac{1}{2} n}\right)= \\
=|z-y|^{2 m-n-|k|}+|z-y|^{2 m-\frac{1}{2} n-|k|}(R(x, y, z))^{\frac{1}{2} n} \preceq \\
\preceq|z-y|^{2 m-n-|k|}+|z-y|^{2 m-\frac{1}{2} n-|k|}|x-z|^{-\frac{1}{2} n} \preceq \\
\preceq|z-y|^{2 m-n-|k|}+|x-z|^{2 m-n-|k|} .
\end{gathered}
$$

In the last step we used that $2 m-n-|k| \leq-\frac{1}{2} n$.

\section{Positivity for related equations and systems}

\subsection{More general equations}

Consider the equation

$$
\left\{\begin{aligned}
\left((-\Delta)^{m}+\mathcal{A}\right) u & =f \text { in } B, \\
\mathcal{D}_{m} u & =0 \text { on } \partial B,
\end{aligned}\right.
$$

with

$$
\mathcal{A}=\sum_{|\alpha|<2 m} a_{\alpha}(x) D^{\alpha}
$$

and $a_{\alpha} \in C(\bar{B})$. The boundary condition is as in (2). The operator $\mathcal{A}$ is a lower order perturbation of $(-\Delta)^{m}$.

In this section we will fix some $p>1$. The operator $\mathcal{G}_{m, n}$ is well defined on $L^{p}(B)$ and we may use the regularity theory of [1].

Theorem 5.1 There exists $\varepsilon_{0}>0$ such that, if $\left\|a_{\alpha}\right\|_{\infty} \leq \varepsilon_{0}$ for all $\alpha$ with $|\alpha|<2 m$, then the following holds.

i. For all $f \in L^{p}(B)$ there exists a solution $u \in W^{2 m, p}(\Omega) \cap W_{0}^{m, p}(\Omega)$ of (43).

ii. Moreover, if $f \in L^{p}(B)$ and $0 \not \equiv f \geq 0$ in $B$, then the solution of (43) satisfies $u>0$ in $B$. 
Remark 1. Let $\Omega$ be a simply connected two dimensional domain and let $h: B \rightarrow \Omega$ be a bijection such that $h\left(x_{1}+i x_{2}\right)=h_{1}\left(x_{1}, x_{2}\right)+i h_{2}\left(x_{1}, x_{2}\right)$ is a holomorphic mapping. Then $\Delta(u \circ h)=\frac{1}{2}|\nabla h|^{2}(\Delta u) \circ h$. We write $g(x)=2|(\nabla h)(x)|^{-2}$. If $\partial \Omega$ is sufficiently smooth, then a Theorem of Kellogg-Warschawski (see [20]) implies that $h$ is sufficiently smooth and that there exist $c_{i}>0$ such that $c_{1} \leq|(\nabla h)(x)| \leq c_{2}$. The elliptic problem $(-\Delta)^{m} u=f$ in $\Omega$ with Dirichlet boundary condition can be transformed to

$$
(-g(\cdot) \Delta)^{m}(u \circ h)=f \circ h \quad \text { in } B
$$

which can also be written as

$$
\left((-\Delta)^{m}+\mathcal{A}\right)(u \circ h)=g^{-m}(f \circ h) \text { in } B,
$$

for some $\mathcal{A}$ as in (44). Since $0 \not \equiv f \geq 0$ yields $0 \not \equiv g^{-m}(f \circ h) \geq 0$ one finds that domains that are close to the disk still have a positive Green function $G_{m, n, \Omega}$. Close to the disk means $\|h-\mathcal{I}\|_{C^{2 m-1}(\bar{B})}$ sufficiently small. For example this holds for an ellipse that is close to a circle, see [12].

The theorem will be proven in two steps. We may rewrite (43) as

$$
u=-\mathcal{G}_{m, n} \mathcal{A} u+\mathcal{G}_{m, n} f
$$

and then formally

$$
u=\left(\mathcal{I}+\mathcal{G}_{m, n} \mathcal{A}\right)^{-1} \mathcal{G}_{m, n} f .
$$

First we will justify that $\left(\mathcal{I}+\mathcal{G}_{m, n} \mathcal{A}\right)^{-1}$ is well defined. The next step will be to prove the estimate $\left(\mathcal{I}+\mathcal{G}_{m, n} \mathcal{A}\right)^{-1} \mathcal{G}_{m, n} \geq c \mathcal{G}_{m, n}$.

Definition 5.2 By $\mathcal{S} \geq \mathcal{T}$ we mean that $(\mathcal{S} f)(x) \geq(\mathcal{T} f)(x)$ for all $f \in L^{p}(B)$ with $f \geq 0$.

Lemma 5.3 Set $W_{D}^{2 m, p}(B):=W^{2 m, p}(B) \cap W_{0}^{m, p}(B)$. If $\left\|a_{\alpha}\right\|_{\infty} \leq \eta_{1}$ and $\eta_{1}$ is sufficiently small, then

$$
\left(\mathcal{I}+\mathcal{G}_{m, n} \mathcal{A}\right)^{-1} \in L\left(W_{D}^{2 m, p}(B) ; W_{D}^{2 m, p}(B)\right) .
$$

Proof. It is sufficient to prove that $\left\|\mathcal{G}_{m, n} \mathcal{A}\right\|<1$. Set

$$
\kappa_{n, m}=\sum_{|k|<2 m} 1=\left(\begin{array}{c}
2 m-1+n \\
2 m-1
\end{array}\right)
$$

and we obtain that

$$
\|\mathcal{A} u\|_{p} \leq \sum_{|\alpha|<2 m}\left\|a_{\alpha}\right\|_{\infty}\left\|D^{\alpha} u\right\|_{p} \leq \kappa_{n, m} \eta_{1}\|u\|_{W^{2 m, p}}
$$

By [1] it follows that $\mathcal{G}_{m, n} \mathcal{A} u \in W_{D}^{2 m, p}(B)$ and that $\mathcal{G}_{m, n}: L^{p}(B) \rightarrow W_{D}^{2 m, p}(B)$ is bounded. For $\eta_{1}$ small enough we have $\left\|\mathcal{G}_{m, n} \mathcal{A}\right\|<1$. 
Lemma 5.4 Let $M$ be as Corollary 4.2, $\left\|a_{\alpha}\right\|_{\infty} \leq \eta$. Then

$$
\mathcal{G}_{m, n} \mathcal{A G}_{m, n} \leq M \eta \kappa_{n, m} \mathcal{G}_{m, n}
$$

with $\kappa_{n, m}$ as in (45).

The proof is straightforward from Corollary 4.2. By symmetry we also have

$$
-\mathcal{G}_{m, n} \mathcal{A G}_{m, n} \leq M \eta \kappa_{n, m} \mathcal{G}_{m, n} .
$$

Lemma 5.5 If $\left\|a_{\alpha}\right\|_{\infty} \leq \eta_{2}$ and $\eta_{2}$ is sufficiently small, then there is $c>0$ such that

$$
\left(\mathcal{I}+\mathcal{G}_{m, n} \mathcal{A}\right)^{-1} \mathcal{G}_{m, n} \geq c \mathcal{G}_{m, n}
$$

Proof of Lemma 5.5. Assume that $f \in L^{p}(B)$. By [1] it follows that $\mathcal{G}_{m, n} f \in$ $W_{D}^{2 m, p}(B)$. The solution $u$ of $(43)$ is the limit in $W_{D}^{2 m, p}(B)$ of $\left\{u_{\nu}\right\}$ where

$$
u_{\nu}=\sum_{i=0}^{\nu}\left(-\mathcal{G}_{m, n} \mathcal{A}\right)^{i} \mathcal{G}_{m, n} f .
$$

Hence there is a subsequence, again denoted by $\left\{u_{\nu}\right\}$, such that $u_{\nu} \rightarrow u$ pointwise. Let us denote $\mathcal{A}_{z}=\sum_{|\alpha|<2 m} a_{\alpha}(z) D_{z}^{\alpha}$ and $G=G_{m, n}$. By Lemma 5.4 we find, assuming $\left\|a_{\alpha}\right\|_{\infty} \leq \eta$, that for $f \geq 0$

$$
\begin{aligned}
& \left|\left(\left(\mathcal{G}_{m, n} \mathcal{A}\right)^{i} \mathcal{G}_{m, n} f\right)(x)\right|= \\
& =\left|\int_{z_{1}} G\left(x, z_{1}\right) \mathcal{A}_{z_{1}} \int_{z_{2}} G\left(z_{1}, z_{2}\right) \mathcal{A}_{z_{2}} \ldots \int_{y} G\left(z_{i}, y\right) f(y) d y d z_{i} \ldots d z_{2} d z_{1}\right|= \\
& =\mid \int_{y} \int_{z_{i}} \ldots \int_{z_{2}} \int_{z_{1}} \frac{G\left(x, z_{1}\right) \mathcal{A}_{z_{1}} G\left(z_{1}, z_{2}\right)}{G\left(x, z_{2}\right)} \frac{G\left(x, z_{2}\right) \mathcal{A}_{z_{2}} G\left(z_{2}, z_{3}\right)}{G\left(x, z_{3}\right)} \ldots \\
& \ldots \frac{G\left(x, z_{i}\right) \mathcal{A}_{z_{i}} G\left(z_{i}, y\right)}{G(x, y)} G(x, y) f(y) d z_{1} d z_{2} \ldots d z_{i} d y \mid \leq \\
& \leq \int_{y} \int_{z_{i}} \ldots \int_{z_{2}} \int_{z_{1}}\left|\frac{G\left(x, z_{1}\right) \mathcal{A}_{z_{1}} G\left(z_{1}, z_{2}\right)}{G\left(x, z_{2}\right)}\right| d z_{1}\left|\frac{G\left(x, z_{2}\right) \mathcal{A}_{z_{2}} G\left(z_{2}, z_{3}\right)}{G\left(x, z_{3}\right)}\right| d z_{2} \ldots \\
& \ldots\left|\frac{G\left(x, z_{i}\right) \mathcal{A}_{z_{i}} G\left(z_{i}, y\right)}{G(x, y)}\right| d z_{i} G(x, y) f(y) d y \leq \\
& \leq\left(\kappa_{n, m} \eta M\right)^{i}\left(\int_{y} G(x, y) f(y) d y\right) \text {. }
\end{aligned}
$$


Take $\eta_{1}$ such that $C=\kappa_{n, m} \eta_{1} M<1$ and it follows from (46) that

$$
\begin{gathered}
\left|u_{\nu}(x)-\left(\mathcal{G}_{m, n} f\right)(x)\right| \leq\left|\left(\sum_{i=1}^{\nu}\left(-\mathcal{G}_{m, n} \mathcal{A}\right)^{i} \mathcal{G}_{m, n} f\right)(x)\right| \leq \\
\leq \sum_{i=1}^{\nu}\left|\left(\left(\mathcal{G}_{m, n} \mathcal{A}\right)^{i} \mathcal{G}_{m, n} f\right)(x)\right| \leq \\
\leq \frac{C}{1-C}\left(\mathcal{G}_{m, n} f\right)(x)
\end{gathered}
$$

which implies

$$
u_{\nu}(x) \geq \frac{1-2 C}{1-C}\left(\mathcal{G}_{m, n} f\right)(x) .
$$

Hence we find for $0 \leq f \not \equiv 0$ that $u_{\nu}>0$ in $B$ for $C<\frac{1}{2}$. Since $C$ does not depend on $\nu$ it follows that $u$ satisfies (48).

Proof of Theorem 5.1. For $\varepsilon_{0}=\eta_{1}$ as in Lemma 5.3 it follows that we have a solution in $W_{D}^{2 m, p}(\Omega)$ of (43) for all $f \in L^{p}(\Omega)$. Indeed, $\mathcal{G}_{m, n} f \in W_{D}^{2 m, p}(\Omega)$ by [1] and Lemma 5.3 implies $u=\left(\mathcal{I}+\mathcal{G}_{m, n} \mathcal{A}\right)^{-1} \mathcal{G}_{m, n} f \in W_{D}^{2 m, p}(\Omega)$. For $\varepsilon_{0}=\eta_{2}\left(<\eta_{1}\right)$ as in Lemma 5.5 we find that $u \geq c \mathcal{G}_{m, n} f>0$.

\subsection{Systems with the same polyharmonic operator}

Consider the system

$$
\left\{\begin{array}{lr}
\mathcal{L} u=\mathcal{A} u+(f, 0, \ldots, 0)^{T} & \text { in } B \\
\mathcal{D} u=0 & \text { on } \partial B
\end{array}\right.
$$

with $u$ a vector-function with $k$ components and where we have

i. $\mathcal{L}$ is a diagonal $k \times k$-matrix of polyharmonic operators: $(L)_{i i}=(-\Delta)^{m}$;

ii. $\mathcal{D}$ is a diagonal $k \times k$-matrix of the corresponding Dirichlet boundary condition: $(\mathcal{D})_{i i}=\mathcal{D}_{m}$

iii. $\mathcal{A}$ is a (full) matrix of lower order coupling terms:

$$
\mathcal{A}_{i, j}=\sum_{|\alpha|<2 m} a_{\alpha}^{i j} D^{\alpha}
$$

with $a_{\alpha}^{i j} \in C(\bar{B})$.

The question is the following. What kind of (small) nonzero coupling is allowed such that one has $u_{1}>0$ whenever $0 \not \equiv f \geq 0$ ?

The last equation of (49), $(-\Delta)^{m} u_{k}=\sum_{j=1}^{k} \mathcal{A}_{k, j} u_{j}$, is 'solved' by

$$
u_{k}=\mathcal{T}_{k} \sum_{j=1}^{k-1} \mathcal{A}_{k, j} u_{j}
$$

where

$$
\mathcal{T}_{k}=\left(\mathcal{I}-\mathcal{G}_{m} \mathcal{A}_{k, k}\right)^{-1} \mathcal{G}_{m}
$$


The operator is $\mathcal{T}_{k}$ is well defined for $\left\|a_{\alpha}^{k k}\right\|_{\infty}$ small. Moreover, for every $\varepsilon>0$ there is $\delta>0$ such that if $\left\|a_{\alpha}^{k k}\right\|_{\infty}<\delta$ then

$$
(1-\varepsilon) \mathcal{G}_{m} \leq \mathcal{T}_{k} \leq(1+\varepsilon) \mathcal{G}_{m} .
$$

We obtain a system with $k-1$ terms, of which the coupling terms are

$$
\mathcal{A}_{i, j}^{(k-1)}=\mathcal{A}_{i, j}+\mathcal{A}_{i, k} \mathcal{T}_{k} \mathcal{A}_{k, j}
$$

Repeating such an argument for the last equation of the newly obtained system, which corresponds to the one but last equation of (49), we find

$$
u_{k-1}=\mathcal{T}_{k-1} \sum_{j=1}^{k-2} \mathcal{A}_{k-1, j}^{(k-1)} u_{j}
$$

where

$$
\mathcal{T}_{k-1}=\left(\mathcal{I}-\mathcal{G}_{m} \mathcal{A}_{k-1, k-1}^{(k-1)}\right)^{-1} \mathcal{G}_{m}
$$

The operator is $\mathcal{T}_{k-1}$ is well defined for $\left\|a_{\alpha}^{k k}\right\|_{\infty},\left\|a_{\alpha}^{k, k-1}\right\|_{\infty},\left\|a_{\alpha}^{k-1, k}\right\|_{\infty}$ and $\left\|a_{\alpha}^{k-1, k-1}\right\|_{\infty}$ small. Moreover, for every $\varepsilon>0$ there is $\delta>0$ such that if $\left\|a_{\alpha}^{i j}\right\|_{\infty}<\delta$ for $i, j \in\{k-1, k\}$ then

$$
(1-\varepsilon) \mathcal{G}_{m} \leq \mathcal{T}_{k-1} \leq(1+\varepsilon) \mathcal{G}_{m}
$$

Etc. In an iterative way one defines $\mathcal{A}^{(k)}=\mathcal{A}$ for $\ell=k$ and for $\ell \in\{1, \ldots, k-1\}$ :

$$
\begin{aligned}
& \mathcal{T}_{\ell}=\left(\mathcal{I}-\mathcal{G}_{m} \mathcal{A}_{\ell, \ell}^{(\ell)}\right)^{-1} \mathcal{G}_{m} \\
& \mathcal{A}_{i, j}^{(\ell-1)}=\mathcal{A}_{i, j}^{(\ell)}+\mathcal{A}_{i, \ell}^{(\ell)} \mathcal{T}_{\ell} \mathcal{A}_{\ell, j}^{(\ell)} \quad 1 \leq i, j \leq \ell-1 .
\end{aligned}
$$

Finally we obtain

$$
u_{1}=\mathcal{T}_{1} f
$$

One proves the following.

Lemma 5.6 If all $\left\|a_{\alpha}^{i j}\right\|_{\infty}$ are sufficiently small, then we find that the operator $\mathcal{T}_{1} \in$ $L\left(L^{p}(B) ; W_{D}^{2 m, p}(B)\right)$ is well defined. Moreover, for all $\varepsilon>0$ there is $\delta>0$, such that if $\left\|a_{\alpha}^{i j}\right\|_{\infty}<\delta$ for all $i, j \leq k,|\alpha|<2 m$, then

$$
(1-\varepsilon) \mathcal{G}_{m} \leq \mathcal{T}_{1} \leq(1+\varepsilon) \mathcal{G}_{m}
$$

The next theorem is a direct corollary.

Theorem 5.7 There exists $\varepsilon_{0}>0$ such that, if $\left\|a_{\alpha}^{i j}\right\|_{\infty} \leq \varepsilon_{0}$ for all $i, j \leq k$ and all $\alpha$ with $|\alpha|<2 m$, then the following holds. If $f \in L^{p}(\Omega)$ with $0 \not \equiv f \geq 0$ in $B$, then the solution of (49) satisfies $u_{1}>0$ in $B$. 


\subsection{Systems with different polyharmonic operators}

In general there is no hope to find

$$
\mathcal{G}_{m, n} \mathcal{A} \mathcal{G}_{\ell, n} \leq c \mathcal{G}_{m, n}
$$

for arbitrary $m$ and $\ell$. However, if $m \leq \ell$ such a result holds.

Lemma 5.8 Suppose $\ell \geq m>0,|k|<2 \ell$. Then we have on $B \times B \times B$ :

$$
\frac{G_{m, n}(x, z)\left|D_{z}^{k} G_{\ell, n}(z, y)\right|}{G_{m, n}(x, y)} \preceq|x-z|^{1-n}+|y-z|^{1-n} .
$$

Proof. We use Proposition 2.4 in the following weak form

$$
\begin{gathered}
\left|D_{z}^{k} G_{\ell, n}(z, y)\right| \preceq|z-y|^{1-n}\left(1 \wedge \frac{d(y)^{\ell}}{|z-y|^{\ell}}\right) \preceq \\
\preceq|z-y|^{1-n}\left(1 \wedge \frac{d(y)^{m}}{|z-y|^{m}}\right)=|z-y|^{2 m-n-(2 m-1)}\left(1 \wedge \frac{d(y)^{m}}{|z-y|^{m}}\right) .
\end{gathered}
$$

Now we may use the proof of Proposition 4.1 replacing $\left|D_{z}^{k} G_{\ell, n}(z, y)\right|$ formally by $\left|D_{z}^{\tilde{k}} G_{m, n}(z, y)\right|$ for some $\tilde{k},|\tilde{k}|=2 m-1$.

Corollary 5.9 Suppose that $\ell \geq m>0$ and let $\mathcal{A}=\sum_{|\alpha|<2 \ell} a_{\alpha} D^{\alpha}$ with $a_{\alpha} \in C(\bar{B})$. Then there is $c>0$ such that (50) holds.

This allows us to generalize the result of the previous section.

Theorem 5.10 Consider (49) with $(L)_{i i}=(-\Delta)^{m_{i}}$ and $(\mathcal{D})_{i i}=\mathcal{D}_{m_{i}}$ and suppose that $m=m_{1} \leq m_{i}$ for all $i \geq 1$. Assume that $\mathcal{A}_{i, j}=\sum_{|\alpha|<2 m_{j}} a_{\alpha}^{i j} D^{\alpha}$. There exists $\varepsilon_{0}>0$ such that, if $\left\|a_{\alpha}^{i j}\right\|_{\infty} \leq \varepsilon_{0}$ for all $i, j$ and $\alpha$, then the following holds. If $f \in L^{p}(\Omega)$ with $0 \not \equiv f \geq 0$ in $B$, then the solution of (49) satisfies $u_{1}>0$ in $B$.

Notice that polyharmonic equations with some non Dirichlet boundary conditions can be taken care of this way. For instance the boundary value problem

$$
\left\{\begin{aligned}
(-\Delta)^{m_{1}+m_{2}+\ldots+m_{k}} u & =f \text { in } B, \\
\mathcal{D}_{m_{1}} \Delta^{m_{2}+m_{3}+\ldots+m_{k}} u & =0 \text { on } \partial B \\
& \vdots \\
\mathcal{D}_{m_{k-2}} \Delta^{m_{k-1}+m_{k}} u & =0 \text { on } \partial B \\
\mathcal{D}_{m_{k-1}} \Delta^{m_{k}} u & =0 \text { on } \partial B \\
\mathcal{D}_{m_{k}} u & =0 \text { on } \partial B
\end{aligned}\right.
$$

can be transformed to a system as in (49) with different polyharmonic operators on the diagonal. 


\section{Only small perturbations}

For second order boundary value problems the following holds. Let $u \in W_{D}^{2, p}(\Omega)$ satisfy

$$
\begin{cases}-\Delta u=a u+f & \text { in } \Omega, \\ u=0 & \text { on } \partial \Omega,\end{cases}
$$

where $\Omega$ is a bounded sufficiently regular domain in $\mathbb{R}^{n}$. Then there exists a first eigenvalue $\lambda_{1}$ such that

- if $a<\lambda_{1}$ then $0 \not \equiv f \geq 0$ implies $u>0$;

- if $a=\lambda_{1}$ then $0 \not \equiv f \geq 0$ implies nonexistence of a solution $u$;

- if $a>\lambda_{1}$ then $0 \not \equiv f \geq 0$ implies $u \nsupseteq 0$.

For the Dirichlet problem with the Laplacian replaced by a polyharmonic operator in general domains there is no such structure. What will remain is the following. Consider

$$
\begin{cases}(-\Delta)^{m} u=a u+f & \text { in } \Omega, \\ \mathcal{D}_{m} u=0 & \text { on } \partial \Omega,\end{cases}
$$

with $\Omega$ a bounded domain in $\mathbb{R}^{n}$ with $\partial \Omega \in C^{2 m, \gamma}$ for some $\gamma>0$. Let $\lambda_{1, m, n}$ denote the first eigenvalue:

$$
\begin{aligned}
\lambda_{1, m, n} & =\inf _{u \in W_{0}^{m, 2}(\Omega)} \frac{\int_{\Omega}\left|\nabla \Delta^{\frac{m-1}{2}} u(x)\right|^{2} d x}{\int_{\Omega}|u(x)|^{2} d x} \text { for } m \text { odd; } \\
\lambda_{1, m, n} & =\inf _{u \in W_{0}^{m, 2}(\Omega)} \frac{\int_{\Omega}\left|\Delta^{\frac{m}{2}} u(x)\right|^{2} d x}{\int_{\Omega}|u(x)|^{2} d x} \text { for } m \text { even. }
\end{aligned}
$$

Let $\Phi_{1, m, n}$ denote a corresponding eigenfunction which is normalized by

$$
\max _{x \in \Omega} \Phi_{1, m, n}(x)=1 .
$$

To simplify notation let $\left((-\Delta)^{m}-a\right)^{i n v}$ denote the Green operator corresponding to the Dirichlet problem (52): $u=\left((-\Delta)^{m}-a\right)^{i n v} f$. These operators

$$
\left((-\Delta)^{m}-a\right)^{i n v}: L^{p}(\Omega) \rightarrow W_{D}^{2 m, p}(\Omega)
$$

are well defined for $a \in C(\bar{\Omega})$ with $a<\lambda_{1, m, n}$.

Theorem 6.1 Suppose that there is $a \in C(\bar{\Omega})$ with $a<\lambda_{1, m, n}$ in $\bar{\Omega}$, such that

$$
\left((-\Delta)^{m}-a\right)^{i n v}: L^{p}(\Omega) \rightarrow W_{D}^{2 m, p}(\Omega) \subset L^{p}(\Omega) \text { is positivity preserving. }
$$

Then for all $b \in C(\bar{\Omega})$ with $a \leq b<\lambda_{1, m, n}$ in $\bar{\Omega}$, we find that

$$
\left((-\Delta)^{m}-b\right)^{i n v}: L^{p}(\Omega) \rightarrow W_{D}^{2 m, p}(\Omega) \subset L^{p}(\Omega) \text { is positivity preserving. }
$$

Moreover, $\left((-\Delta)^{m}-a\right)^{i n v}$ being strongly positive implies $\left((-\Delta)^{m}-b\right)^{i n v}$ to be strongly positive. 
Remark 1. An operator $T: L^{p}(\Omega) \rightarrow L^{p}(\Omega)$ is called strongly positive iff $0 \not \equiv f \geq 0$ implies $T f>0$.

Lemma 6.2 Let $\lambda<\lambda_{1, m, n}$ and suppose that $\left((-\Delta)^{m}-\lambda\right)^{i n v}: L^{p}(\Omega) \rightarrow W_{D}^{2 m, p}(\Omega) \subset$ $L^{p}(\Omega)$ is strongly positive. Then $\Phi_{1, m, n}$ is unique and $\Phi_{1, m, n}>0$.

Remark 2. If $\left((-\Delta)^{m}-\lambda\right)^{i n v}$ can be represented by an integral operator, then this lemma is a consequence of a theorem by Jenč (see [21, page 337]).

Lemma 6.3 Let $m>1$. For $\lambda \ll 0$ the operator $\left((-\Delta)^{m}-\lambda\right)^{i n v}: L^{p}(\Omega) \rightarrow$ $W_{D}^{2 m, p}(\Omega) \subset L^{p}(\Omega)$ is not positivity preserving.

Remark 3. The result in Lemma 6.3 seems to be folklore, as we learnt from Bernis [3], but we have not been able to locate a reference for this fact.

In the following corollary we will suppose that $u \in W_{D}^{2 m, p}(\Omega)$ and $f \in L^{p}(\Omega)$ are as in (52).

Corollary 6.4 Let $m>1$ and suppose that $\Phi_{1, m, n}>0$. Then there is $-\infty<\lambda_{c} \leq$ $\lambda_{1, m, n}$, depending on $m, n$ and $\Omega$, such that for all $a \in C(\bar{\Omega})$ the following holds.

i. If $a<\lambda_{c}$, then

$$
0 \not \equiv f \geq 0 \Rightarrow u \not \leq 0,
$$

but not positivity preserving:

$$
\begin{aligned}
& \exists 0 \not \equiv f \geq 0 \text { with } u \geq 0 ; \\
& \exists 0 \not \equiv f \geq 0 \text { with } u \nsupseteq 0 .
\end{aligned}
$$

ii. If $\lambda_{c} \leq a<\lambda_{1, m, n}$, then positivity preserving:

$$
f \geq 0 \Rightarrow u \geq 0 \text {. }
$$

iii. If $a=\lambda_{1, m, n}$, then for $0 \not \equiv f \geq 0$ there is no solution.

iv. If $a>\lambda_{1, m, n}$, then positivity killing:

$$
0 \not \equiv f \geq 0 \Rightarrow u \nsupseteq 0 .
$$

Remark 4. If $m=1$ then by standard results we find that the same holds with $\lambda_{c}=-\infty$.

Remark 5. The fact that $\left((-\Delta)^{m}-\lambda\right)^{i n v}$ is not positivity preserving for $\lambda \ll 0$ implies that the corresponding parabolic initial boundary value problem doesn't preserve positive initial values. See also [2].

Remark 6. By the example in [17] one finds that for $m>1$ a first eigenfunction $\Phi_{1, m, n}$ may change sign in strictly convex, arbitrarily smooth domains. If $\Omega$ is the unit ball then $\Phi_{1, m, n}>0$. We expect $\Phi_{1, m, n}$ to be positive on domains that are close to a ball. Such a result holds for $n=2$, see [12]. 
Proof of Corollary 6.4. First we prove iv. Let $a>\lambda_{1, m, n}$ and suppose that we have $0 \not \equiv f \geq 0$ and $0 \not \equiv u \geq 0$. Then, like in the case of the Laplacian:

$$
\begin{gathered}
0<\int_{\Omega} f \Phi_{1, m, n} d x=\int_{\Omega}\left((-\Delta)^{m} u-a u\right) \Phi_{1, m, n} d x= \\
=\int_{\Omega} u\left(\lambda_{1, m, n}-a\right) \Phi_{1, m, n} d x \leq 0
\end{gathered}
$$

which is a contradiction.

iii. also follows by (53).

ii. From Theorem 6.1 one finds that the set of $\lambda$ in $\mathbb{R}$ for which the operator $\left((-\Delta)^{m}-\lambda\right)^{i n v}: L^{p}(\Omega) \rightarrow W_{D}^{2 m, p}(\Omega) \subset L^{p}(\Omega)$ is positivity preserving is an interval $\left[\lambda_{c}, \lambda_{1, m, n}\right)$ or $\left(\lambda_{c}, \lambda_{1, m, n}\right)$. Lemma 6.3 yields that $\lambda_{c}>-\infty$ when $m>1$. Since

$$
\left(\lambda \mapsto\left((-\Delta)^{m}-\lambda\right)^{i n v}\right): \mathbb{R} \rightarrow L\left(L^{p}(\Omega) ; W_{D}^{2 m, p}(\Omega)\right)
$$

is continuous except at the eigenvalues, we find that the interval is left closed.

i. The first claim again follows by (53). From the previous argument we find that $\left((-\Delta)^{m}-\lambda\right)^{i n v}$ is not positivity preserving for any $\lambda<\lambda_{c}$. Now suppose that $a \leq \lambda<\lambda_{c}$. If $\left((-\Delta)^{m}-a\right)^{i n v}$ is positivity preserving then by Theorem 6.1 $\left((-\Delta)^{m}-\lambda\right)^{i n v}$ would be positivity preserving, which is a contradiction. The other claim follows by taking $u=\Phi_{1, m, n}$. Indeed, $\Phi_{1, m, n}>0$ and $\left((-\Delta)^{m}-a\right) \Phi_{1, m, n}=$ $\left(\lambda_{1, m, n}-a\right) \Phi_{1, m, n}>0$.

Proof of Lemma 6.2. First some preliminaries that can be found in [21]. $(E, \leq)$ is a vector lattice if $f, g \in E$ implies $f \vee g, f \wedge g \in E$. $(E,\|\cdot\|, \leq)$ is called a Banach lattice if $(E,\|\cdot\|)$ is a Banach space and if $(E, \leq)$ is a vector lattice such that $|f| \leq|g|$ implies $\|f\| \leq\|g\|$. A subset $A \subset E$ is called a lattice ideal if $|f| \leq|g|$ and $g \in A$ implies $f \in A$. An operator $S$ on a Banach lattice $E$ is called irreducible if $\{0\}$ and $E$ are the only closed lattice ideals that are invariant under $S$.

One has that $L^{p}(\Omega)$ is a Banach lattice and that the operator

$$
T:=\left((-\Delta)^{m}-\lambda\right)^{i n v}: L^{p}(\Omega) \rightarrow W_{D}^{2 m, p}(\Omega) \subset L^{p}(\Omega)
$$

is positive, compact and irreducible. By De Pagter's Theorem ([19]) the spectral radius of $T$ is positive. By the Krein-Rutman Theorem (see e.g. [21]) it follows that the first eigenvalue of $T$ has a unique (up to multiplication) eigenfunction $\Phi$ and that $\Phi>0$. Since its first eigenvalue is $\left(\lambda_{1, m, n}-\lambda\right)^{-1}$ we find that $\Phi$ satisfies $(-\Delta)^{m} \Phi=\lambda_{1, m, n} \Phi$ and hence that $\Phi_{1, m, n}=c \Phi$ and $\Phi_{1, m, n}>0$.

Proof of Lemma 6.3. We have to show that $\left((-\Delta)^{m}-\lambda\right)^{i n v}$ is not positivity preserving for $\lambda \ll 0$. We use different arguments than Bernis in [3].

If $\left((-\Delta)^{m}\right)^{i n v}$ is not positivity preserving we are done. Hence we may assume that $\left((-\Delta)^{m}\right)^{i n v}$ does preserve positivity. We construct a function $a \in C(\bar{\Omega})$, with $a \leq 0$ such that $\left((-\Delta)^{m}-a\right)^{i n v}$ is not positivity preserving.

Set $u=\left((-\Delta)^{m}\right)^{i n v} 1$, which is hence positive by assumption. Since $u \in W_{D}^{2 m, n}(\Omega) \subset$ $C(\bar{\Omega})$ there will be some $B_{\rho}\left(x_{0}\right) \subset \Omega$ such that $u \geq \varepsilon>0$ in $\overline{B_{\rho}\left(x_{0}\right)}$. Let $\chi \in C^{\infty}(\mathbb{R})$ be such that $0 \leq \chi \leq 1, \chi(r)=0$ for $r>1$ and $\chi=1$ in $r<\frac{1}{2}$. We define

$$
\tilde{u}(x)=\chi\left(\frac{\left|x-x_{0}\right|}{\rho}\right)\left(16 \rho^{-2}\left|x-x_{0}\right|^{2}-1\right)+\left(1-\chi\left(\frac{\left|x-x_{0}\right|}{\rho}\right)\right) u(x) .
$$


Then the following holds: there exists some $\delta \in \mathbb{R}^{+}$such that

$$
(-\Delta)^{m} \tilde{u}\left\{\begin{array}{l}
=0 \quad \text { for }\left|x-x_{0}\right| \leq \frac{1}{2} \rho, \\
\geq-\delta^{-1} \text { for } \frac{1}{2} \rho \leq\left|x-x_{0}\right|<\rho, \\
=1 \quad \text { elsewhere in } \Omega,
\end{array}\right.
$$

and

$$
\tilde{u}\left\{\begin{array}{l}
<0 \text { for }\left|x-x_{0}\right|<\frac{1}{4} \rho \\
\geq 0 \text { for } \frac{1}{4} \rho \leq\left|x-x_{0}\right|<\frac{1}{2} \rho \\
\geq \delta \text { for } \frac{1}{2} \rho \leq\left|x-x_{0}\right|<\rho \\
\geq 0 \text { elsewhere in } \Omega .
\end{array}\right.
$$

Next we define

$$
a(x)=-\delta^{-2}\left(1-\chi\left(2 \frac{\left|x-x_{0}\right|}{\rho}\right)\right)
$$

and we find that

$$
\left((-\Delta)^{m}-a\right) \tilde{u}\left\{\begin{array}{lll}
= & 0 & \text { for }\left|x-x_{0}\right|<\frac{1}{4} \rho, \\
\geq & 0 & \text { for } \frac{1}{4} \rho \leq\left|x-x_{0}\right|<\frac{1}{2} \rho, \\
\geq & -\delta^{-1}+\delta^{-2} \delta & \text { for } \frac{1}{2} \rho \leq\left|x-x_{0}\right|<\rho, \\
\geq & 1 & \text { elsewhere in } \Omega .
\end{array}\right.
$$

We constructed a sign-changing function $\tilde{u}$ with $\left((-\Delta)^{m}-a\right) \tilde{u} \geq 0$ in $\Omega$ and $\mathcal{D}_{m} \tilde{u}=$ 0 on $\partial \Omega$. Hence Theorem 6.1 yields that $\left((-\Delta)^{m}-\lambda\right)^{i n v}$ is not positivity preserving for $\lambda \leq-\delta^{-2}=\min a$.

Proof of Theorem 6.1. First assume that $p \geq 2$. Suppose that $u \in W_{D}^{2 m, p}$ satisfies

$$
(-\Delta)^{m} u-b u=f \text { in } \Omega,
$$

and we find that $u$ solves

$$
(-\Delta)^{m} u-a u=f+(b-a) u \text { in } \Omega,
$$

where $(b-a) \geq 0$.

We will show that the nonlinear problem

$$
\left\{\begin{array}{lr}
\left((-\Delta)^{m}-a\right) u=f+(b-a)|u| & \text { in } \Omega, \\
\mathcal{D}_{m} u=0 & \text { on } \partial \Omega,
\end{array}\right.
$$

has a solution $u_{0}$. If a solution $u_{0}$ exists we will find that $f+(b-a)\left|u_{0}\right| \geq 0$ implies

$$
u_{0}=\left((-\Delta)^{m}-a\right)^{i n v}\left(f+(b-a)\left|u_{0}\right|\right) \geq 0 .
$$

The existence of $u_{0}$ is established by minimizing the functional $F$ on $W_{0}^{m, 2}(\Omega)$ defined by

$$
F(u)=\frac{1}{2} \int_{\Omega}\left(|\Psi u|^{2}-a u^{2}-2 f u-(b-a)|u| u\right) d x,
$$

where

$$
\Psi u=\left\{\begin{array}{cc}
\Delta^{\frac{m}{2}} u & \text { if } m \text { is even } \\
\nabla \Delta^{\frac{m-1}{2}} u & \text { if } m \text { is odd }
\end{array}\right.
$$


We prove that $F$ is coercive on $W_{0}^{m, 2}(\Omega)$. Set

$$
\varepsilon=2 \lambda_{1, m, n} \wedge \min _{x \in \bar{\Omega}}\left(\lambda_{1, m, n}-b(x)\right) .
$$

Then

$$
\begin{gathered}
F(u) \geq \frac{1}{2} \int_{\Omega}\left(|\Psi u|^{2}-a u^{2}-2 f u-(b-a) u^{2}\right) d x \geq \\
\geq \frac{1}{2} \int_{\Omega}\left(\left(\frac{\frac{1}{2} \varepsilon}{\lambda_{1, m, n}}+\frac{\lambda_{1, m, n}-\frac{1}{2} \varepsilon}{\lambda_{1, m, n}}\right)|\Psi u|^{2}-b u^{2}-2 f u\right) d x \geq \\
\geq \frac{1}{2} \int_{\Omega}\left(\frac{\varepsilon}{2 \lambda_{1, m, n}}|\Psi u|^{2}+\left(\lambda_{1, m, n}-\frac{1}{2} \varepsilon-b\right) u^{2}-2 f u\right) d x \geq \\
\geq \frac{1}{2} \int_{\Omega}\left(\frac{\varepsilon}{2 \lambda_{1, m, n}}|\Psi u|^{2}+\frac{1}{2} \varepsilon u^{2}-2 f u\right) d x \geq \\
\geq \frac{\varepsilon}{4 \lambda_{1, m, n}} \int_{\Omega}|\Psi u|^{2} d x-\frac{1}{\varepsilon} \int_{\Omega} f^{2} d x .
\end{gathered}
$$

Now let $\left\{u_{\nu}\right\}_{\nu=1}^{\infty}$ be a minimizing sequence in $W_{0}^{m, 2}(\Omega)$. Since $F(\cdot)$ is sequentially weakly lower semicontinuous and coercive in $W_{0}^{m, 2}(\Omega)$ there exists a weak limit in $W_{0}^{m, 2}(\Omega)$, say $u_{0}$, and $F\left(u_{0}\right) \leq \liminf _{\nu \rightarrow \infty} F\left(u_{\nu}\right)$. Since $u_{\nu} \rightarrow u_{0}$ in $W_{0}^{m, 2}(\Omega)$ we find $u_{\nu} \rightarrow u_{0}$ in $L^{2}(\Omega)$. Using regularity theory and appropriate imbeddings a bootstrapping argument shows that $u_{0} \in W_{D}^{2 m, p}(\Omega)$. As we remarked before we find that $u_{0} \geq 0$ and hence that $u_{0}$ solves (52) with $b$ instead of $a$. For $p \in(1,2)$ the claim follows by approximating $f \in L^{p}(\Omega)$ by $f_{k} \in L^{2}(\Omega)$ and using [1].

Acknowledgements This work was supported by the Deutsche Forschungsgemeinschaft.

\section{References}

1. S. Agmon, A. Douglis and L. Nirenberg, Estimates near the boundary for solutions of elliptic partial differential equations satisfying general boundary conditions I, Commun. Pure Appl. Math. 12, 623-727 (1959)

2. F. Bernis, Change of sign of the solutions to some parabolic problems, in: V. Lakshmikantham (ed.), Nonlinear Analysis and Applications, pp. 75-82, New York: Marcel Dekker 1987

3. F. Bernis, private communication

4. T. Boggio, Sulle funzioni di Green d'ordine m, Rend. Circ. Mat. Palermo 20, 97-135 (1905)

5. M. Cranston, E.Fabes and Z. Zhao, Conditional gauge and potential theory for the Schrödinger operator, Transactions Amer. Math. Soc. 307, 171-194 (1988)

6. R.J. Duffin, On a question of Hadamard concerning super-biharmonic functions, J. Math. Phys. 27, 253-258 (1949)

7. P.R. Garabedian, Partial Differential Equations, second edition, New York: Chelsea 1986

8. D. Gilbarg and N.S. Trudinger, Elliptic Partial Differential Equations of Second Order, second edition, Berlin Heidelberg New York: Springer-Verlag 1983

9. H.-Ch. Grunau, Das Dirichletproblem für semilineare elliptische Differentialgleichungen höherer Ordnung, Thesis, Göttingen 1990

10. H.-Ch. Grunau, The Dirichlet problem for some semilinear elliptic differential equations of arbitrary order, Analysis 11, 83-90 (1991)

11. H.-Ch. Grunau and G. Sweers, Classical solutions for some higher order semilinear elliptic equations under weak growth conditions, Nonlinear Anal., to appear 
12. H.-Ch. Grunau and G. Sweers, Positivity for perturbations of polyharmonic operators with Dirichlet boundary conditions in two dimensions, Math. Nachr., to appear

13. M. Grüter and K.O. Widman, The Green function for uniformly elliptic equations, Manuscr. Math. 37, 303-342 (1982)

14. W. Gunsenheimer, Nichtnegativität Greenscher Funktionen für $u^{(i v)}+u=f$ in Abhängigkeit vom zugrundeliegenden Definitionsbereich $[-L, L]$, Arbeitsbericht, Universität Bayreuth, 1994

15. I.W. Herbst and Z. Zhao, Note on a $3 G$ theorem $(d=2)$, in: E. Çinlar e.a. (eds.), Seminar on Stochastic Processes, 1988, pp. 183-184, Boston Basel Berlin: Birkhäuser 1989

16. H. Hueber and M. Sieveking, Uniform bounds for the quotients of Green functions on $C^{1,1}$ domains, Ann. Inst. Fourier 32, 105-117 (1982)

17. V.A. Kozlov, V.A. Kondrat'ev and V.G. Maz'ya, On sign variation and the absence of "strong" zeros of solutions of elliptic equations, Math. USSR Izvestiya 34, 337-353 (1990)

18. E. Mitidieri and G. Sweers, Weakly coupled elliptic systems and positivity, Math. Nachr. 173, 259-286 (1995)

19. B. de Pagter, Irreducible compact operators, Math. Z. 192, 149-153 (1986)

20. Ch. Pommerenke, Boundary behaviour of conformal maps, Berlin Heidelberg New York: Springer 1992

21. H.H. Schaefer, Banach lattices and positive operators, Berlin Heidelberg New York: Springer 1974

22. H.S. Shapiro and M. Tegmark, An elementary proof that the biharmonic Green function of an eccentric ellipse changes sign, SIAM Rev. 36, 99-101 (1994)

23. G. Sweers, Positivity in $C(\bar{\Omega})$ for elliptic systems, Math. Z. 209, 251-271 (1992)

24. G. Sweers, Positivity for a strongly coupled elliptic systems by Green function estimates, J. Geometric Analysis 4, 121-142 (1994)

25. K.O. Widman, Inequalities for the Green function and boundary continuity of the gradient of solutions of elliptic differential equations, Math. Scand. 21, 17-37 (1967)

26. Z. Zhao, Green function for Schrödinger operator and conditioned Feynman-Kac gauge, J. Math. Anal. Appl. 116, 309-334 (1986)

27. Z. Zhao, Green functions and conditioned gauge theorem for a 2-dimensional domain, in: E. Çinlar e.a. (eds.), Seminar on Stochastic Processes, 1987, pp. 283-294, Boston Basel Berlin: Birkhäuser 1988 